\title{
FOURIER APPROACH TO HOMOGENIZATION PROBLEMS
}

\author{
${\text { Carlos } \text { Conch }^{1} \text { and M. VAnninathan }}^{2}$
}

\begin{abstract}
This article is divided into two chapters. The classical problem of homogenization of elliptic operators with periodically oscillating coefficients is revisited in the first chapter. Following a Fourier approach, we discuss some of the basic issues of the subject: main convergence theorem, Bloch approximation, estimates on second order derivatives, correctors for the medium, and so on. The second chapter is devoted to the discussion of some non-classical behaviour of vibration problems of periodic structures.
\end{abstract}

Mathematics Subject Classification. 35B27, 35A25, 42C30.

Received January 14, 2002.

\section{INTRODUCTION}

Asymptotic methods in Partial Differential Equations form an important part of the contributions of Professor J.-L. Lions to Applied Mathematics. He devoted a significant part of his research activities to apply these methods to many problems of interest. Among these, Homogenization was his pet subject for quite sometime. His book on the subject [8] written with his collaborators is regarded by many as a master piece and cited widely. We feel honored to contribute this article which is concerned with one of his favorite themes. Further, Professor J.-L. Lions was instrumental in shaping our scientific career. Taking this opportunity, we place on record our sincere gratitude and appreciation and dedicate this article in his memory. The contents are divided into two chapters: 1 . Stationary problems and 2. Vibration problems. These can be read independently except for the Section 1.5 which is needed in Chapter 2 also.

This article presents a Fourier approach to understand homogenization of periodic structures. The aim here is to state the main results with motivations, highlight various phenomena in the Fourier space and indicate possible gains over other methods. Main ideas are given avoiding technicalities. The paper is essentially based on authors' publications with their collaborators and also on some current on-going unpublished works. The compiled bibliography is vast but obviously not complete.

\footnotetext{
Keywords and phrases: Homogenization, Bloch waves, correctors, regularity, spectral problems, vibration problems.

${ }_{1}^{1}$ Departamento de Ingeniería Matemática, and Centro de Modelamiento Matemático, Universidad de Chile, Casilla 170/3, Correo-3, Santiago, Chile; e-mail: cconca@dim.uchile.cl

2 IISc-TIFR Mathematics Programme, TIFR Centre, P.O. Box 1234, Bangalore 560 012, India;

e-mail: vanni@math.tifrbng.res.in
} 


\section{Stationary PROBlems}

\subsection{A homogenization problem}

Let us consider a family of inhomogeneous media occupying a certain bounded region $\Omega$ in $\mathbb{R}^{N}$, parameterized by a small parameter $\varepsilon$ and represented by $N \times N$ matrices of real-valued functions $a^{\varepsilon}(x)=\left[a_{k l}^{\varepsilon}(x)\right]$ defined on $\Omega$. The positive parameter $\varepsilon$ defines a length scale measuring how densely inhomogeneities are distributed in $\Omega$. As concrete examples, we can think of a mixture of heat conducting materials with different conductivities or composite elastic materials with different stiffness coefficients. In such cases, $a^{\varepsilon}(x)$ is the matrix of conductivities or stiffness of the material present at $x \in \Omega$. As $\varepsilon \rightarrow 0$, one is thus in the presence of micro-structures, namely, an arrangement of these materials, which makes the medium highly heterogeneous. Regarding the behaviour of these individual materials, we assume that they obey Fourier's law or Hooke's law as the case may be. This motivates us to consider the following boundary value problem in the general case:

$$
\left\{\begin{array}{l}
A^{\varepsilon} u^{\varepsilon}(x) \stackrel{\text { def }}{=}-\frac{\partial}{\partial x_{k}}\left(a_{k l}^{\varepsilon}(x) \frac{\partial u^{\varepsilon}}{\partial x_{l}}(x)\right)=f(x), \quad x \in \Omega \\
u^{\varepsilon}=0 \text { on } \partial \Omega
\end{array}\right.
$$

(The usual summation convention is followed throughout.) In this write-up, we will be concerned with a special type of micro-structures, namely periodic structures which are defined by

$$
a_{k l}^{\varepsilon}(x)=a_{k l}\left(\frac{x}{\varepsilon}\right), \quad k, l=1, \ldots N .
$$

Here $a_{k l}=a_{k l}(y)$ are functions on $\mathbb{R}^{N}$ which are $Y$-periodic where we take $\left.Y=\right] 0,2 \pi\left[{ }^{N}\right.$ to be the basic cell. In the sequel, we reserve the symbol \# to denote periodicity property.

Next, we introduce some non-degeneracy assumptions on the materials so that the above problem is wellposed:

$$
\left\{\begin{array}{l}
a_{k l} \in L_{\#}^{\infty}(Y) \forall k, l=1, \ldots, N, \\
a(y)=\left[a_{k l}(y)\right] \text { is a symmetric matrix, } \\
\text { For some } 0<\alpha<\beta, \quad \alpha|\eta|^{2} \leq a_{k l}(y) \eta_{l} \eta_{k} \leq \beta|\eta|^{2} \quad \forall \eta \in \mathbb{R}^{N}, y \in \mathbb{R}^{N} .
\end{array}\right.
$$

Above structural hypotheses ensure that for $f \in L^{2}(\Omega)$, there is a unique solution $u^{\varepsilon}$ in $H^{1}(\Omega)$ such that $\left\|u^{\varepsilon}\right\|_{H^{1}} \leq C$ where $H^{1}(\Omega)$ is the usual Sobolev space:

$$
H^{1}=\left\{v \in L^{2} ; \quad \frac{\partial v}{\partial x_{j}} \in L^{2} \quad \forall j=1, \ldots N\right\}
$$

The main difficulty lies not with the existence question of the solution but with its numerical computation. Typically as $\varepsilon \rightarrow 0$, the solution develops oscillations on fine length scale of order $\varepsilon$ indicating that, on the Fourier side, high wave numbers acquire more energy. As a consequence, direct numerical simulations are rendered difficult. What is the way out? One way is to average out the high wave number components of the solution and incorporate their effects on low wave number components. To carry this out, a natural idea is to consider weak limits $u^{*}$ of $\left\{u^{\varepsilon}\right\}$ in $H^{1}$ which provide a computable approximation to $u^{\varepsilon}$ for $\varepsilon$ small. To make this idea work, we must have an independent characterization of $u^{*}$. This amounts to finding the constitutive law of the mixture which is one of the aims of Homogenization Theory (see $[8,12,35,43])$.

\subsection{Methods of Homogenization}

It is fair to say that ever since the publication of the book [8], there is a renewed and vigorous activity in homogenization problems which form an important area of Applied Mathematics. Several aspects of the class of 
problems introduced in the previous section have been studied, and new classes of problems and new questions were generated from the application side. In order to tackle these questions, several methods have been devised and there is an enormous literature on the subject. Our modest goal in this part is to address certain basic questions which we consider to be fundamental.

Methods introduced so far can be broadly categorized as follows: (A) Physical space methods, (B) Fourier space methods, and (C) Phase space methods. There are many methods falling in the class (A) and let us mention some successful ones: Multiscale expansion [8], Multiscale convergence [2,37], Method of oscillating test functions [35,47], Compensated Compactness [36], Gamma Convergence [23] and so on. Though they have varying domains of applicability, they have some common characteristics. An insight into the kind of oscillations produced by the medium is gained and this information is used in some way or the other (ansatz, test functions, ...).

In order to study PDEs with variable coefficients, techniques of Pseudo-Differential Operators and Fourier Integral Operators were developed [30]. These phase space theories provide tools suitable to study the behaviour of inhomogeneous media which are qualitatively similar to homogeneous ones and their small perturbations. To deal with heterogeneities possessing qualitatively different properties, we need more sophisticated tools such as $H$-measures [46], micro-local defect measures [27,28], Wigner measures [29,32] and their generalizations and refinements (see [34]).

In the middle ground between the classes $(\mathrm{A})$ and $(\mathrm{C})$ lie the methods based purely on Fourier techniques and these notes address them. In problems involving oscillations, it is natural to seek methods based on Fourier space and this general outlook led us to look for tools suitable to describe oscillations produced by heterogeneities in the Fourier space. Our intention is to present some of them in this write-up and illustrate how they can be effectively used to study the problem at hand. If the medium is homogeneous, Fourier techniques have proved to be extremely useful both analytically and numerically. In the same way, these techniques, if extended properly to non-homogeneous cases, are expected to be as fruitful.

\subsection{Issues discussed}

Though there are many aspects of homogenization, we will confine ourselves to some of them which we consider to be fundamental. Using the tools of Fourier space, one simple objective here to shed new light and offer an alternate way to view old results. Since these tools are sharp, we are also able to bring out certain new features and results which are not easily obtainable by other means. This should attract the attention of homogenizers. Multiscale structure of the solution, if any, is a consequence and not an a priori assumption. Passage to the limit is more direct and there is no need for sophisticated test functions. The way the test functions arise in this approach is also pointed out. Following points are discussed in this chapter:

(i) existence of the homogenization limit;

(ii) when the limit exists, it will provide zero $^{\text {th }}$ order approximation to the solution $u^{\varepsilon}$ and to the nonhomogeneous medium represented by $a^{\varepsilon}(x)$. What about higher order approximations called correctors? In this context, we introduce a new object called the Bloch approximation. While the notion of correctors for the solution $u^{\varepsilon}$ is old, the corresponding notion for the medium appears to be new. This possibility is due to the Fourier approach that we follow;

(iii) it was pointed out earlier that the first order derivatives of $u^{\varepsilon}$ are bounded. Under what condition, do we have uniform estimates on the second order derivatives? This aspect is new and appears not discussed elsewhere.

These points will be elaborated later in the respective sections. For the moment, let us indicate how the method proceeds.

\subsection{Description of the method and plan of the chapter}

As is well-known, there are difficulties in treating boundaries in Fourier analysis, and so we begin by considering our equation in the entire space. The next step is to decompose the solution into small parts and this is called Bloch decomposition presented in the next section. We will need two types of decomposition. One among 
them is the spectral decomposition of the operator $A^{\varepsilon}$ in $L^{2}\left(\mathbb{R}^{N}\right)$ in terms of its eigenvalues and eigenvectors called Bloch waves. Using these decompositions, we can transform, in an equivalent way, our questions from the physical space to Bloch space. In the homogenization process, only the component corresponding to the first Bloch wave (called ground state) matters; higher Bloch modes are not excited and hence they are negligible. This is shown in Section 1.6. The advantage of working in the Fourier space is thus clear: instead of considering the operator $A^{\varepsilon}$, it is enough to consider the scalar, namely the ground eigenvalue. Regarding the ground state, we prove in Section 1.9 that it converges to the familiar plane wave (or Fourier wave) in the homogenization limit. This is an indication of the fact that periodically heterogeneous medium approximates homogeneous medium. The corresponding eigenvalue (called ground energy) is shown to tend to the homogenized operator written in the Fourier space (see Sect. 1.10). These results require regularity properties of the ground energy and the ground state. We show (in Sect. 1.7) that these elements are indeed analytic for small momenta. In Section 1.8, we indicate a systematic method of calculating their derivatives and give their physical space interpretation. Somewhat surprisingly, the usual Taylor expansion thus obtained yields homogenization and corrector results (see Sects. 1.11, 1.12). This is another effect of working with Fourier approach. Finally in Section 1.13, we discuss uniform estimates on the second order derivatives of $u^{\varepsilon}$ using the second Bloch decomposition that we mentioned earlier.

\subsection{Bloch decomposition}

To motivate things to come, let us consider the case where the coefficients $a_{k l}(y)=\delta_{k l} \forall k, l$. Here the operator involved is $(-\Delta)$ which defines an unbounded self-adjoint operator in $L^{2}\left(\mathbb{R}^{N}\right)$ with domain $H^{2}\left(\mathbb{R}^{N}\right)$. In the sequel, this case is referred to as the homogeneous case. It is known that its spectrum is $[0, \infty)$ and its spectral resolution is provided by the generalized eigenbasis formed by plane waves (also called Fourier waves) $\mathrm{e}^{i x \cdot \xi}, \xi \in \mathbb{R}^{N}$. The operator itself acts as a multiplier:

$$
-\Delta\left(\mathrm{e}^{i x \cdot \xi}\right)=|\xi|^{2} \mathrm{e}^{i x \cdot \xi}, \quad \xi \in \mathbb{R}^{N}
$$

We want to generalize the above picture to the periodic medium represented by

$$
A \stackrel{\text { def }}{=}-\frac{\partial}{\partial y_{k}}\left(a_{k l}(y) \frac{\partial}{\partial y_{l}}\right), \quad y \in \mathbb{R}^{N} .
$$

The counter-part of plane waves are Bloch waves which were originally introduced in Solid State Physics in the context of propagation of electrons in a crystals, see [9]. Additional references are [8, 26, 38, 42, 44, 49]. These waves are obtained from the plane waves via scattering by the periodic medium. This means that they are generalized eigenvectors of $A$ (as in the homogeneous case); however, we must impose a scattering condition which was implicitly satisfied in the homogeneous case.

Definition. Fix a momentum vector $\eta \in \mathbb{R}^{N}$. Consider the eigenvalue problem: find $\lambda=\lambda(\eta) \in \mathbb{C}$ and $\psi(\eta)=\psi(y, \eta)$ (non-zero) such that

$$
\begin{gathered}
A \psi=\lambda \psi \text { in } \mathbb{R}^{N}, \psi \text { is }(\eta, Y) \text { - periodic, } \\
\text { i.e., } \psi(y+2 \pi p)=\mathrm{e}^{2 \pi i p \cdot \eta} \psi(y), \quad p \in \mathbb{Z}^{N}, y \in \mathbb{R}^{N} .
\end{gathered}
$$

The eigenvectors $\psi$ are known as Bloch waves associated with $A$ and the eigenvalues $\lambda$ are called Bloch eigenvalues.

The above periodicity condition is non-standard in homogenization. When $\eta=0$, it coincides with the usual $Y$-periodicity which is very familiar. This periodicity condition can be motivated in a number of ways. Perhaps 
the quickest way to motivate it is to consider the case when $\eta$ is rational. A very natural class of vibrations of the periodic structure is defined by the following eigenvalue problem:

$$
A \psi_{n}=\lambda_{n} \psi_{n} \text { in } \mathbb{R}^{N}, \quad \psi_{n} \text { is } Y_{n} \text { - periodic, }
$$

where $Y_{n}$ is the rectangle $] 0,2 \pi n\left[{ }^{N}\right.$ with $n=\left(n_{1}, n_{2}, \ldots, n_{N}\right)$ being a positive multi-integer. In the usual homogenization theory, we are used to consider periodic oscillations with period $Y$. The above problem describes more general oscillations, namely, those of multiple cells. In fact, there are $n_{1} n_{2} \cdots n_{N}$ cells in the rectangle $Y_{n}$ and they can exhibit independent oscillations not necessarily of $Y$-periodic type. However, this problem can be reduced to an eigenvalue problem on the basic cell $Y$ but with a different type of periodic condition. To obtain this, let us note the following intuitive orthogonal decomposition invariant under $A$ :

$$
L_{\#}^{2}\left(Y_{n}\right)=\bigoplus_{\omega} L_{\#}^{2}(\omega, Y)
$$

where

$$
L_{\#}^{2}(\omega, Y)=\left\{\psi \in L_{\mathrm{loc}}^{2}\left(\mathbb{R}^{N}\right) ; \psi(y+2 \pi p)=\omega^{p} \psi(y) \quad \forall p \in \mathbb{Z}^{N}, y \in \mathbb{R}^{N}\right\},
$$

and where $\omega \in S^{1} \times S^{1} \times \cdots \times S^{1}$ is such that the $j^{\text {th }}$ component of $\omega$ varies among all the $n_{j}^{\text {th }}$ roots of unity. Necessarily, such $\omega$ 's are of the form $\left(\mathrm{e}^{2 \pi i \eta_{1}}, \ldots, \mathrm{e}^{2 \pi i \eta_{N}}\right)$ where $\eta_{1}, \eta_{2}, \ldots, \eta_{N}$ are rationals. With this notation, we note that the above space consists of $(\eta, Y)$-periodic functions. Any irrational $\eta$ can be approximated by rationals by varying $n$ and noting that the set of roots of unity is dense in $S^{1}$. For details, see [1] and [17].

The above Bloch eigenvalue problem is invariant if $\eta$ is replaced by $\eta+l, l \in \mathbb{Z}^{N}$. Hence, without loss of generality, we can restrict $\eta$ to the cell $\left.Y^{\prime}=\right]-1 / 2,1 / 2\left[{ }^{N}\right.$, called the reciprocal cell. This result is expected on physical grounds too. Indeed, it is heuristically clear that the medium produces oscillations up to unit length scales and hence a localization on the unit cell is expected on the Fourier side.

Periodic media in one dimension were studied by Floquet [25] prior to Bloch. The above eigenvalue problem can be solved using his ansatz that $\psi(y, \eta)=\mathrm{e}^{i y \cdot \eta} \phi(y, \eta)$; indeed the problem is transformed to another one where the parameter $\eta$ appears in the operator rather in the boundary condition:

$$
A(\eta) \phi=\lambda(\eta) \phi \quad \text { in } \quad \mathbb{R}^{N}, \quad \phi \text { is } Y \text {-periodic. }
$$

Here $A(\eta)$ is the so-called shifted operator

$$
A(\eta) \stackrel{\text { def }}{=}-\left(\frac{\partial}{\partial y_{k}}+i \eta_{k}\right)\left[a_{k l}(y)\left(\frac{\partial}{\partial y_{l}}+i \eta_{l}\right)\right] .
$$

In the usual manner, we can solve the above problem in the space

$$
H_{\#}^{1}(Y)=\left\{v \in H_{\mathrm{loc}}^{1}\left(\mathbb{R}^{N}\right) ; v \text { is } Y \text { - periodic }\right\} .
$$

This is because the bilinear form associated with $A(\eta)$, viz

$$
a(\eta)(u, v)=\int_{Y} a_{k l}(y)\left(\frac{\partial u}{\partial y_{l}}+i \eta_{l} u\right)\left(\overline{\frac{\partial v}{\partial y_{k}}+i \eta_{k} v}\right) \mathrm{d} y
$$

is coercive on this space. To this end, we note that a kind of Poincaré inequality is valid. More precisely, we have

$$
c\left\{\|\nabla v\|_{0, Y}+|\eta|\|v\|_{0, Y}\right\} \leq\|\nabla v+i \eta v\|_{0, Y} \leq\left\{\|\nabla v\|_{0, Y}+|\eta|\|v\|_{0, Y}\right\}
$$


for all $v \in H_{\#}^{1}(Y)$ and $\eta \in Y^{\prime}$. The conclusion of the preceding arguments is the following description of the scattered waves for a fixed momentum:

Theorem 1.1. Fix $\eta \in Y^{\prime}$. Then there exist a sequence of real eigenvalues $\left\{\lambda_{m}(\eta) ; m \in \mathbb{N}\right\}$ and corresponding eigenvectors $\left\{\phi_{m}(y, \eta) ; m \in \mathbb{N}\right\}$ such that

(i) $A(\eta) \phi_{m}(y, \eta)=\lambda_{m}(\eta) \phi_{m}(y, \eta) \quad \forall m \in \mathbb{N}$;

(ii) $0 \leq \lambda_{1}(\eta) \leq \lambda_{2}(\eta) \leq \ldots \rightarrow \infty$. Each eigenvalue is of finite multiplicity;

(iii) $\left\{\phi_{m}(\cdot, \eta) ; m \in \mathbb{N}\right\}$ is an orthonormal basis for $L_{\#}^{2}(Y)$.

If we now vary the momentum vector $\eta \in Y^{\prime}$, we expect to get the required spectral resolution of $A$ in $L^{2}\left(\mathbb{R}^{N}\right)$. More precisely, we have

(i) $\left\{\mathrm{e}^{i y \cdot \eta} \phi_{m}(y, \eta) ; m \in \mathbb{N}, \eta \in Y^{\prime}\right\}$ forms a basis of $L^{2}\left(\mathbb{R}^{N}\right)$ in a generalized sense;

(ii) As a consequence, $L^{2}\left(\mathbb{R}^{N}\right)$ can be identified with $L^{2}\left(Y^{\prime} ; l^{2}(\mathbb{N})\right)$;

(iii) $A$ acts as a multiplication operator: $A\left(\mathrm{e}^{i y \cdot \eta} \phi_{m}(y, \eta)\right)=\lambda_{m}(\eta) \mathrm{e}^{i y \cdot \eta} \phi_{m}(y, \eta)$.

The above heuristic arguments show that the spectrum of $A$, denoted by $\sigma(A)$, coincides with the so-called Bloch spectrum which is defined as the union of the images of all the mappings $\lambda_{m}(\cdot)$, i.e.,

$$
\sigma(A)=\sigma_{\text {Bloch }} \stackrel{\text { def }}{=} \bigcup_{m=1}^{\infty}\left[\inf _{\eta \in Y^{\prime}} \lambda_{m}(\eta), \sup _{\eta \in Y^{\prime}} \lambda_{m}(\eta)\right] .
$$

We thus see a band structure of the spectrum. Unlike the homogeneous case, $\sigma(A)$ needs not fill up the entire $\mathbb{R}^{+}$and there may be gaps, see [24]; however, we expect that these gaps are closed in the homogenization limit. The precise result stated next is a generalization of an analogous result in Fourier transform:

Theorem 1.2 (Bloch Decomposition). Let $g \in L^{2}\left(\mathbb{R}^{N}\right)$. Define $m^{\text {th }}$ Bloch coefficient of $g$ by

$$
\left(B_{m} g\right)(\eta)=\int_{\mathbb{R}^{N}} g(y) \mathrm{e}^{-i y \cdot \eta} \bar{\phi}_{m}(y, \eta) \mathrm{d} y, \quad m \in \mathbb{N}, \eta \in Y^{\prime} .
$$

Then the following inverse formula holds:

$$
g(y)=\int_{Y^{\prime}} \sum_{m=1}^{\infty}\left(B_{m} g\right)(\eta) \mathrm{e}^{i y \cdot \eta} \phi_{m}(y, \eta) \mathrm{d} \eta, \quad y \in Y .
$$

Further, we have Parseval's equality:

$$
\int_{\mathbb{R}^{N}}|g(y)|^{2} \mathrm{~d} y=\int_{Y^{\prime}} \sum_{m=1}^{\infty}\left|B_{m} g(\eta)\right|^{2} \mathrm{~d} \eta .
$$

In order to deduce the above result from Theorem 1.1, we must express an arbitrary $g \in L^{2}\left(\mathbb{R}^{N}\right)$ as a linear superposition with respect to $\eta \in Y^{\prime}$ of $(\eta, Y)$-periodic functions. This is done by means of periodization technique.

Theorem 1.3. For $g \in L^{2}\left(\mathbb{R}^{N}\right)$, we define

$$
g_{\#}(y, \eta)=\sum_{p \in \mathbb{Z}^{N}} g(y+2 \pi p) \mathrm{e}^{-i(y+2 \pi p) \cdot \eta} .
$$

Then we have

(i) $g_{\#}(y, \eta)$ is $Y$-periodic w.r.t. y; equivalently, $g_{\#}(y, \eta) \mathrm{e}^{i y \cdot \eta}$ is $(\eta, Y)$-periodic w.r.t. $y$; 
(ii) $g_{\#}(y, \eta) \mathrm{e}^{i y \cdot \eta}$ is $Y^{\prime}-$ periodic w.r.t. $\eta$;

(iii) the following inversion holds:

$$
g(y)=\int_{Y^{\prime}} g_{\#}(y, \eta) \mathrm{e}^{i y \cdot \eta} \mathrm{d} \eta, \quad y \in \mathbb{R}^{N}
$$

(iv) Parseval equality is valid:

$$
\begin{gathered}
\int_{Y^{\prime}}\left|g_{\#}(y, \eta)\right|^{2} \mathrm{~d} \eta=\sum_{p \in \mathbb{Z}^{N}}|g(y+2 \pi p)|^{2}, \quad y \in \mathbb{R}^{N}, \\
\int_{Y} \int_{Y^{\prime}}\left|g_{\#}(y, \eta)\right|^{2} \mathrm{~d} \eta \mathrm{d} y=\int_{\mathbb{R}^{N}}|g(y)|^{2} \mathrm{~d} y .
\end{gathered}
$$

Bloch waves at $\varepsilon$-scale. Following the steps indicated above, we can introduce spectral decomposition of $A^{\varepsilon}$ for each $\varepsilon>0$. The spectral elements are denoted by $\left\{\lambda_{m}^{\varepsilon}(\xi), \phi_{m}^{\varepsilon}(x, \xi) ; m \geq 1, \xi \in \varepsilon^{-1} Y^{\prime}\right\}$. The corresponding Bloch transform is denoted by $B_{m}^{\varepsilon} g(\xi)$. Since the operators $A$ and $A^{\varepsilon}$ are related by the change of variables $y=\frac{x}{\varepsilon}$, we have simple relations between the corresponding Fourier variables and the spectral elements:

$$
\lambda_{m}^{\varepsilon}(\xi)=\varepsilon^{-2} \lambda_{m}(\eta), \quad \phi_{m}^{\varepsilon}(x, \xi)=\phi_{m}(y, \eta), \quad y=\frac{x}{\varepsilon}, \quad \eta=\varepsilon \xi
$$

The appearance of the phase variable $\varepsilon \xi$ is not a surprise if we recall the relation $\widehat{g\left(\frac{x}{\varepsilon}\right)}(\xi)=\varepsilon^{N} \hat{g}(\varepsilon \xi)$ in Fourier transform.

\subsection{Higher Bloch modes are negligible}

Thanks to the Bloch decomposition, we can transform our differential equation $A^{\varepsilon} u^{\varepsilon}(x)=f(x), x \in \mathbb{R}^{N}$ to a set of algebraic equations

$$
\lambda_{m}^{\varepsilon}(\xi) B_{m}^{\varepsilon} u^{\varepsilon}(\xi)=B_{m}^{\varepsilon} f(\xi), \quad \xi \in \varepsilon^{-1} Y^{\prime}, m \geq 1
$$

We will now see why the equations corresponding to $m \geq 2$ can be neglected in the homogenization process. We remark that the discrete indexing parameter $m \in \mathbb{N}$ is due to the ellipticity of the system and for the same reason, we have what we call spectral separation. More precisely, we have the estimate which is a consequence of min-max principle:

$$
\inf \left\{\lambda_{m}(\eta) ; \eta \in Y^{\prime}, m \geq 2\right\} \geq \lambda_{2}^{(N)}>0,
$$

where $\lambda_{2}^{(N)}$ is the second eigenvalue of the spectral problem for $A$ in the cell with Neumann boundary condition. As far as the first Bloch eigenvalue is concerned, our results below will show that

$$
C_{1}|\eta|^{2} \leq \lambda_{1}(\eta) \leq C_{2}|\eta|^{2} \quad \forall \eta \in Y^{\prime}
$$

Thus our terminology spectral separation is justified.

Now, we introduce the projection of $u^{\varepsilon}$ corresponding to all higher Bloch modes:

$$
v^{\varepsilon}(x)=\int_{\varepsilon^{-1} Y^{\prime}} \sum_{m=2}^{\infty} B_{m}^{\varepsilon} u^{\varepsilon}(x) \mathrm{e}^{i x \cdot \xi} \phi_{m}^{\varepsilon}(x, \xi) \mathrm{d} \xi .
$$

Thanks to the spectral separation, we can deduce that (see $[14,20])$.

$$
\left\|v^{\varepsilon}\right\|_{H^{1}\left(\mathbb{R}^{N}\right)} \leq C \varepsilon\|f\|_{L^{2}\left(\mathbb{R}^{N}\right)} \text {, and }\left\|v^{\varepsilon}\right\|_{H^{1}\left(\mathbb{R}^{N}\right)} \leq C \varepsilon^{2}\|f\|_{H^{1}\left(\mathbb{R}^{N}\right)} .
$$


These estimates show that $v^{\varepsilon}$ does not matter in homogenization. Moreover, it can be ignored in the analysis of correctors of first and second order too.

\subsection{Regularity of ground state}

In order to pass to the limit in the remaining equation $\lambda_{1}^{\varepsilon}(\xi) B_{1}^{\varepsilon} u^{\varepsilon}(\xi)=B_{1}^{\varepsilon} f(\xi)$, we need regularity properties of the first Bloch mode $\phi_{1}(y, \eta)$ for small momentum $|\eta|$. It is natural to seek regularity properties of the corresponding eigenvalue and then deduce those of eigenvectors. Even though $A(\eta)$ depends quadratically on $\eta$, we cannot assert that its eigenvalues have the same dependence. However, thanks to min-max principle, we can establish (see [20]):

Proposition 1.4. For $m \geq 1, \lambda_{m}(\eta)$ is a Lipschitz function of $\eta \in Y^{\prime}$.

It is not clear how to carry over this regularity to eigenvectors even around $\eta=0$. We cannot expect global regularity on physical grounds. Indeed, our equation may be taken as a model for the behaviour of certain crystals. From the physics of crystals and their singularities, we do not expect global Lipschitz regularity with respect to $\eta \in Y^{\prime}$.

Our idea to circumvent these difficulties is to do a regular perturbation analysis of the first eigenvalue $\lambda_{1}(\eta)$ of $A(\eta)$ around $\eta=0$. The fact that $\eta$ is a vector parameter rather than a scalar does not allow us to apply the classical results [42] directly. However, the difficulties can be overcome, see [14,20]. The crucial property that we use is the fact that $\lambda_{1}(0)=0$ is a simple eigenvalue of $A(0)=A$; corresponding eigenvectors being constants. We prove

Theorem 1.5. There exists $\delta>0$ such that $\lambda_{1}(\eta)$ is analytic in the ball $B_{\delta}=\{\eta ;|\eta|<\delta\}$ and there is a choice of corresponding unit eigenvector $\phi_{1}(y, \eta)$ satisfying

$$
\begin{gathered}
\eta \rightarrow \phi_{1}(\cdot, \eta) \in H_{\#}^{1}(Y) \text { is analytic on } B_{\delta} ; \phi_{1}(y, 0) \stackrel{\text { def }}{=}|Y|^{-1 / 2} \stackrel{\text { def }}{=}(2 \pi)^{-N / 2} \\
\left\|\phi_{1}(\cdot, \eta)\right\|_{L^{2}(Y)}=1, \quad \operatorname{Im} \int_{Y} \phi_{1}(y, \eta) \mathrm{d} y=0, \quad \eta \in B_{\delta} .
\end{gathered}
$$

To prove analyticity of $\lambda_{1}(\eta)$, we convert the eigenvalue problem to a parameterized algebraic equation $D(\eta, \lambda)$ $=0$ where, heuristically, $D(\eta, \lambda) \equiv \operatorname{det}(\lambda I-A(\eta))$. We can then apply Implicit Function Theorem in the analytic category.

Treatment of eigenvectors is more difficult because unlike eigenvalues, they are not uniquely determined. Even if we impose normalization condition on them, there is still an arbitrary phase factor which has to be chosen properly. That is why we stipulate the somewhat unconventional condition that the average of $\phi_{1}(\cdot, \eta)$ is real. Useful tool in the choice of eigenvectors is Dunford Integral:

$$
P(\eta)=\frac{1}{2 \pi i} \int_{\Gamma_{r}}(A(\eta)-\lambda)^{-1} \mathrm{~d} \lambda
$$

where $\Gamma_{r}$ is the circle $\{\lambda \in \mathbb{C} ;|\lambda|=r\}$ in the complex plane. We choose $r>0$ and $\delta>0$ small enough and independent of $\eta \in B_{\delta}$ in such a way that $\Gamma_{r}$ encloses only the first eigenvalue $\lambda_{1}(\eta)$ of $A(\eta)$ for $\eta \in B_{\delta}$ and all other eigenvalues lie outside. Since $P(\eta)$ is an analytic projection onto the eigenspace of $\lambda_{1}(\eta)$, we can take $P(\eta) \phi_{1}(\cdot, 0)$ as a first choice for eigenvector. This can be modified further to satisfy other requirements. Finally, we mention that a partial regularity result for eigenvalues and eigenvectors can be found in [49] in the case of Schrödinger equation with a potential.

\subsection{Taylor expansion of ground state}

Let us begin with a remark on the homogeneous case where $a_{k l}(y)=\delta_{k l}$. Here one can easily check that the spectrum is indexed by $m \in \mathbb{Z}^{N}$ and $\lambda_{m}(\eta)=|m+\eta|^{2}, \phi_{m}(y, \eta)=\mathrm{e}^{i m \cdot y}$ so that $\lambda_{m}(\eta)$ is a quadratic polynomial 
with respect to $\eta$ and $\phi_{m}$ is independent of $\eta$. These are the effects of homogeneity on the Fourier side. When the medium is heterogeneous, we could establish only local analyticity of $\lambda_{1}(\eta)$ and $\phi_{1}(\cdot, \eta)$ at $\eta=0$.

Having done this, we can, in principle, compute all their derivatives at $\eta=0$ and obtain the Taylor expansion. Somewhat surprisingly, all the derivatives can be expressed in terms of objects computable in the physical space. (This is one place where there is a link between the two approaches). These computations are not easy and the procedure involves repeated differentiation of the eigenvalue relation $A(\eta) \phi_{1}(\cdot, \eta)=\lambda_{1}(\eta) \phi_{1}(\cdot, \eta)$ and evaluation at $\eta=0$. The operations are performed in [14] and we give the principal results here:

(T1) All odd order derivatives of $\lambda_{1}(\eta)$ at $\eta=0$ vanish.

(T2) All odd order derivatives of $\phi_{1}(\cdot, \eta)$ at $\eta=0$ are purely imaginary. For instance, the first order derivatives at $\eta=0$ are given by

$$
\frac{\partial \phi_{1}}{\partial \eta_{k}}(y, 0)=i|Y|^{-1 / 2} \chi_{k}(y)
$$

where $\chi_{k}(y)$ is the unique solution of the cell problem

$$
\left\{\begin{array}{l}
A \chi_{k}=\frac{\partial a_{k l}}{\partial y_{l}} \text { in } \quad \mathbb{R}^{N}, \quad \chi_{k} \in H_{\#}^{1}(Y), \\
M_{Y}\left(\chi_{k}\right) \stackrel{\text { def }}{=} \frac{1}{|Y|} \int_{Y} \chi_{k} \mathrm{~d} y=0 .
\end{array}\right.
$$

(T3) All even order derivatives of $\phi_{1}(\cdot, \eta)$ at $\eta=0$ are real.

(T4) Second order derivatives of $\lambda_{1}(\eta)$ at $\eta=0$ are given by

$$
\frac{1}{2} \frac{\partial^{2} \lambda_{1}}{\partial \eta_{k} \partial \eta_{l}}(0)=q_{k l}, \quad \forall k, l=1, \ldots, N,
$$

where $q_{k l}$ are the so-called homogenized coefficients defined by

$$
q_{k l}=M_{Y}\left(a_{k l}+a_{k n} \frac{\partial \chi_{l}}{\partial y_{n}}\right)
$$

Generally, eigenvectors are not uniquely determined. In the present situation, it is a small surprise to know that the conditions imposed on $\phi_{1}$ in the last section uniquely determine it at least in a neighborhood of $\eta=0$. The property (T1) which amounts to saying that $\lambda_{1}(\eta)$ is an even function on $B_{\delta}$ is not evident from physical space considerations. Jointly (T1) and (T4) give an invariant characterization of the homogenized matrix: it corresponds to the Hessien matrix of $\lambda_{1}(\eta)$ at a critical point.

\subsection{First Bloch transform tends to Fourier transform}

Various Bloch transforms $B_{m}^{\varepsilon} g(\xi), m \geq 1$, are intrinsic quantities associated with our periodic medium and the Fourier transform $\hat{g}(\xi)$ is similarly associated with a homogeneous medium. As already seen, one can ignore $B_{m}^{\varepsilon} g(\xi)$, for $m \geq 2$ in homogenization. Regarding $B_{1}^{\varepsilon} g(\xi)$, we notice that it is defined in terms of the first Bloch wave $\phi_{1}\left(\frac{x}{\varepsilon}, \varepsilon \xi\right) \mathrm{e}^{i x \cdot \xi}$ which converges to the Fourier wave $(2 \pi)^{-N / 2} \mathrm{e}^{i x \cdot \xi}$ because of the continuity of $\phi_{1}(\cdot, \eta)$ at $\eta=0$. Thus $B_{1}^{\varepsilon} g(\xi) \rightarrow \hat{g}(\xi)$. These heuristic arguments can be formalized in a number of ways. As a sample, let us cite from $[14,20]$.

Theorem 1.6. (i) If $g^{\varepsilon} \rightarrow g$ weakly in $L^{2}\left(\mathbb{R}^{N}\right)$, then $\chi_{\varepsilon^{-1} Y^{\prime}} B_{1}^{\varepsilon} g^{\varepsilon} \rightarrow \hat{g}$ weakly in $L_{\mathrm{loc}}^{2}\left(\mathbb{R}^{N}\right)$ provided there is a fixed compact set $K$ such that $\operatorname{supp}\left(g^{\varepsilon}\right) \subset K$ for all $\varepsilon$.

(ii) If $g \in L^{2}\left(\mathbb{R}^{N}\right)$, then $\chi_{\varepsilon^{-1} Y^{\prime}} B_{1}^{\varepsilon} g \rightarrow \hat{g}$ in $L^{2}\left(\mathbb{R}^{N}\right)$. 


\subsection{Main homogenization result}

The way is now clear to state and discuss the principal homogenization result. To start with, we consider the equation in $\mathbb{R}^{N}$ :

$$
\left\{\begin{array}{lll}
u^{\varepsilon} \rightarrow u^{*} & \text { in } & H^{1}\left(\mathbb{R}^{N}\right) \text { weak } \\
A^{\varepsilon} u^{\varepsilon}=f & \text { in } \quad \mathbb{R}^{N}
\end{array}\right.
$$

The question is to know the equation satisfied by $u^{*}$. Fundamental difficulty in the physical space is to identify the limit of the product of two weakly converging sequences. More precisely, consider the heat flux/stress defined by

$$
\sigma_{k}^{\varepsilon}=a_{k l}^{\varepsilon} \frac{\partial u^{\varepsilon}}{\partial x_{l}} \quad \forall k=1, \ldots, N
$$

We know $a_{k l}^{\varepsilon} \rightarrow M_{Y}\left(a_{k l}\right)$ in $L^{\infty}$ weak $^{*}$ and $\frac{\partial u^{\varepsilon}}{\partial x_{l}} \rightarrow \frac{\partial u^{*}}{\partial x_{l}}$ in $L^{2}$ weak. However, we cannot assert that $\sigma_{k}^{\varepsilon} \rightarrow M_{Y}\left(a_{k l}\right) \frac{\partial u^{*}}{\partial x_{l}}$ in $L^{2}$ weak. Thus the constitutive equation governing the limiting material is not clear. This is due to the oscillating nature of sequences. On the Fourier side, these oscillations disappear and we do not see the above difficulties. More precisely, taking the first Bloch transform of the equation, we obtain

$$
\lambda_{1}^{\varepsilon}(\xi) B_{1}^{\varepsilon} u^{\varepsilon}(\xi)=B_{1}^{\varepsilon} f(\xi), \quad \xi \in \varepsilon^{-1} Y^{\prime}
$$

It is straightforward to pass to the limit in this equation using the results established earlier on the Bloch spectrum. We get

$$
q_{k l} \xi_{l} \xi_{k} \hat{u}^{*}(\xi)=\hat{f}(\xi), \quad \xi \in \mathbb{R}^{N}
$$

This is precisely the Fourier space version of the so-called homogenized equation, namely,

$$
A^{*} u^{*} \stackrel{\text { def }}{=}-q_{k l} \frac{\partial^{2} u^{*}}{\partial x_{k} \partial x_{l}}=f(x), \quad x \in \mathbb{R}^{N} .
$$

However, there is a small flaw in our arguments. Since $\left\{u^{\varepsilon}\right\}$ does not have uniform compact support, we cannot directly apply Theorem 1.6. However, this can be overcome by multiplying $u^{\varepsilon}$ by a cut-off function. The same technique enables us to handle the presence of boundaries too. Since the homogenized coefficients do not depend on boundaries, the above result is after all expected. Details are furnished in [20].

Theorem 1.7. Let $\Omega \subset R^{N}$ be an arbitrary domain. Consider a sequence $u^{\varepsilon} \rightarrow u^{*}$ in $H^{1}(\Omega)$ weak and $A^{\varepsilon} u^{\varepsilon}=f$ in $\Omega$ with $f \in L^{2}(\Omega)$. Then $u^{*}$ satisfies $A^{*} u^{*}=f$ in $\Omega$. In fact, $\sigma_{k}^{\varepsilon} \rightarrow q_{k l} \frac{\partial u^{*}}{\partial x_{l}}$ in $L^{2}(\Omega)$ weak.

Before concluding this section, we want to point out one important feature. Our periodic medium is represented by $\left\{\lambda_{m}(\eta) ; \eta \in Y^{\prime}, m \in \mathbb{N}\right\}$ in the basis $\left\{\phi_{m}(y, \eta) ; \eta \in Y^{\prime}, m \in \mathbb{N}\right\}$. However, only a very little of this information is used for homogenization; namely the behaviour of the dominant eigenvalue $\lambda_{1}(\eta)$ for $\eta$ in an arbitrarily small neighborhood of $\eta=0$. This extra localization is an added advantage of working on the Fourier side.

\subsection{Correctors for the solution}

Homogenized limit $u^{*}$ captures only an averaged behaviour of the heterogeneous medium. The exact solution $u^{\varepsilon}$, of course, has deviations/fluctuations around $u^{*}$. How to approximate these? The notion of correctors of various orders is useful in this context. The idea is to look for terms which when added to $u^{*}$ provide a better approximation to $u^{\varepsilon}$. For instance, first order correctors provide an approximation in the energy norm $H^{1}$ there by improving weak convergence. Second order correctors yield an error estimate in the energy norm of 
order $O(\varepsilon)$. Of course, correctors are not unique. First order correctors based on the analysis in physical space (via multiscale expansion) were proposed in [8]. From our experience of working in the Fourier space, we are naturally motivated to suggest, what we call Bloch approximation $\theta^{\varepsilon}$ to the solution $u^{\varepsilon}$. We will see that this object contains both first and second order correctors. Its important feature is that it is easily computable and the computations show that its performance is numerically superior [13].

Unlike the homogenized coefficients, the correctors are influenced by the boundaries and the corresponding analysis is technically complicated [15]. Here, we confine ourselves to the analysis of correctors in the entire space $\mathbb{R}^{N}$. Therefore, we consider an oscillating sequence $\left\{u^{\varepsilon}\right\}$ and $f \in L^{2}\left(\mathbb{R}^{N}\right)$ satisfying

$$
\left\{\begin{array}{l}
A^{\varepsilon} u^{\varepsilon}=f \text { in } \mathbb{R}^{N} \\
u^{\varepsilon} \rightarrow u^{*} \text { in } H^{1}\left(\mathbb{R}^{N}\right) \text { weak } \\
u^{\varepsilon} \rightarrow u^{*} \text { in } L^{2}\left(\mathbb{R}^{N}\right) \text { strong. }
\end{array}\right.
$$

In the sequel, we suggest a corrector for such a sequence. The last condition may raise certain eyebrows, but it is a natural hypothesis to work with. In smooth bounded domains $\Omega \subset \mathbb{R}^{N}$, this condition is a consequence of weak convergence in $H^{1}$ by Rellich's Lemma. However, the same cannot be said in the case of $\mathbb{R}^{N}$ even if we assume $A^{\varepsilon} u^{\varepsilon}=f$ in $\mathbb{R}^{N}$; there can be concentration of $L^{2}$-energy at infinity. That is why, we are explicitly assuming $L^{2}$-strong convergence. Moreover, the natural elliptic operator in $\mathbb{R}^{N}$ to consider is $\left(A^{\varepsilon}+I\right)$ and not $A^{\varepsilon}$. In such case, one can indeed prove that there is no concentration of $L^{2}$-energy at infinity.

It is somewhat surprising but it is true that higher Bloch modes $\left\{\phi_{m}^{\varepsilon}, m \geq 2\right\}$ do not contribute even at the level of correctors. Therefore, we introduce the projection onto the space of the first Bloch mode: for $g \in L^{2}\left(\mathbb{R}^{N}\right)$, we define

$$
P_{1}^{\varepsilon} g(x)=\int_{\varepsilon^{-1} Y^{\prime}} B_{1}^{\varepsilon} g(\xi) \mathrm{e}^{i x \cdot \xi} \phi_{1}^{\varepsilon}(x, \xi) \mathrm{d} \xi, \quad x \in \mathbb{R}^{N} .
$$

Since $B_{1}^{\varepsilon} u^{\varepsilon}$ is close to $\hat{u}^{*}$, it is natural to introduce the Bloch approximation by

$$
\theta^{\varepsilon}(x)=\int_{\varepsilon^{-1} Y^{\prime}} \hat{u}^{*}(\xi) \mathrm{e}^{i x \cdot \xi} \phi_{1}^{\varepsilon}(x, \xi) \mathrm{d} \xi, \quad x \in \mathbb{R}^{N} .
$$

By our construction, $\theta^{\varepsilon}$ is close to $P_{1}^{\varepsilon} u^{\varepsilon}$, and hence to $u^{\varepsilon}$. This can be rigorously established [14].

Theorem 1.8. We have $\left(u^{\varepsilon}-\theta^{\varepsilon}\right) \rightarrow 0$ in $H^{1}\left(\mathbb{R}^{N}\right)$ and $\left|u^{\varepsilon}-\theta^{\varepsilon}\right|_{H^{1}\left(\mathbb{R}^{N}\right)} \leq C \varepsilon\|f\|_{L^{2}\left(\mathbb{R}^{N}\right)}$.

It is worth remarking that corrector results in the literature usually assume additional hypotheses on the regularity of corrector terms. Here, we make no such hypotheses.

If we wish to see the physical space structure of the corrector $\theta^{\varepsilon}$, it is enough to expand $\phi_{1}(y, \eta)$ as a power series in $\eta$ for $|\eta|<\delta$. From the definition of $\theta^{\varepsilon}$, we obtain

$$
\theta^{\varepsilon}(x)=u^{*}(x)+\varepsilon \chi_{k}\left(\frac{x}{\varepsilon}\right) \frac{\partial u^{*}}{\partial x_{k}}(x)+\cdots
$$

The structure of each term in the expansion is very clear. It is the product of two factors: one is the derivative of $u^{*}$ of a certain order and the other one is an oscillating test function. This is similar to the asymptotic expansion of the solution $u^{\varepsilon}$ obtained in [8]. We must however highlight the difference in obtaining them. Our method uses the classical Taylor expansion whereas multiscale ansatz was used in [8]. One sees again how oscillations are replaced by localization. Even though the expansion of $\phi_{1}(\cdot, \eta)$ is valid only for $|\eta|<\delta$, and the integral representation for $\theta^{\varepsilon}$ involves all $\eta \in Y^{\prime}$, above asymptotic expansion up to three terms for $\theta^{\varepsilon}$ can be surprisingly justified throughout $\mathbb{R}^{N}$. This shows that $\theta^{\varepsilon}$ indeed contains at least first and second order correctors. See [14] for details. 


\subsection{Correctors to the medium}

This section briefly reports on the on-going work [16]. Our aim here is to indicate yet another possible advantage with the Fourier approach to Homogenization. Earlier, we have discussed correctors to the solution $u^{\varepsilon}$. In a similar spirit, we now focus on obtaining correctors to the periodic medium itself. More precisely, we regard the homogenized medium as a first approximation and seek to improve upon it. Why such a concept is needed? To answer this, let us consider the propagation of waves in the periodic medium modelled by the wave equation $\frac{\partial^{2}}{\partial t^{2}}+A^{\varepsilon}$. If the propagating wave has a wavelength bigger than the size of the microstructure, namely $\varepsilon$, a dominant feature that occurs after a span of time is dispersion of waves. Indeed, the one-dimensional computations done in [45] show that a single blob develops smaller and smaller tail of blobs demonstrating dispersion phenomenon. The wave equation associated with the homogenized operator, namely $\frac{\partial^{2}}{\partial t^{2}}+A^{*}$ is good to describe propagation up to a certain time but inadequate to describe dispersion because the corresponding dispersion relation is trivial. What is needed to overcome this difficulty is a further approximation to the medium.

It may be possible to explain dispersion by including higher order terms in the multiscale expansion in the physical space for the wave equation. However, it is not clear how to obtain correctors to the medium via these techniques. Fourier space techniques offer a natural framework in this regard. Indeed, let us consider again the Taylor expansion of the first Bloch eigenvalue $\lambda_{1}(\eta)$ which represents the periodic medium:

$$
\lambda_{1}(\eta)=q_{k l} \eta_{k} \eta_{l}+b_{k l m n} \eta_{k} \eta_{l} \eta_{m} \eta_{n}+\cdots
$$

The first term provides the homogenized medium. If we include the second term also, we get a fourth order operator. As the corresponding dispersion relation is not trivial, this offers an exciting possibility to explain dispersion. However, computing the tensor $b_{k l m n}$ (which means computing $4^{\text {th }}$ order derivatives of $\lambda_{1}(\eta)$ at $\eta=0$ ) and analyzing its properties is not easy. In [16] we establish the following:

(i) $b_{k l m n} \eta_{k} \eta_{l} \eta_{m} \eta_{n} \leq 0 \forall \eta \in \mathbb{R}^{N}$. This property, showing that the tensor does not change sign, came as a surprise to us. We do not know whether higher order derivatives enjoy a similar property;

(ii) the tensor $b_{k l m n}$ can be degenerate: there exists a non-constant $a(y)$ and $\eta \neq 0$ such that $b_{k l m n} \eta_{k} \eta_{l} \eta_{m} \eta_{n}$ $=0$;

(iii) in one-dimension, however, such a thing does not happen. More precisely, we have $\lambda_{1}^{(4)}(0)$ is non-positive and it vanishes iff $a(y)$ is a constant.

The implication of these properties on the wave propagation problem is investigated in [16].

\subsection{Uniform estimates on second order derivatives}

The first naive attempt to approximate the operator $A^{\varepsilon}$ in the homogenization process is to replace the rapidly oscillating coefficients $a_{k l}^{\varepsilon}$ by their averages $M_{Y}\left(a_{k l}\right)$ and consider the operator

$$
\bar{A} \stackrel{\text { def }}{=}-\frac{\partial}{\partial x_{k}}\left(M_{Y}\left(a_{k l}\right) \frac{\partial}{\partial x_{l}}\right) .
$$

In one's early education in homogenization, one is taught that even though this is a natural thing to do, this is not the correct way of homogenizing $A^{\varepsilon}$. In this section, we investigate if this attitude is right or wrong. To our surprise, we will see that there are non-trivial materials for which the homogenized operator is indeed $\bar{A}$.

In fact, we address the following stronger question: consider $u^{\varepsilon}$ such that $u^{\varepsilon} \rightarrow u^{*}$ in $H^{1}(\Omega)$ weak and $A^{\varepsilon} u^{\varepsilon}=f i n \Omega$. Under what condition does there exist a constant $C$, independent of $\varepsilon$ such that

$$
\left\|u^{\varepsilon}\right\|_{H_{\mathrm{loc}}^{2}(\Omega)} \leq C\|f\|_{L_{\mathrm{loc}}^{2}(\Omega)} ?
$$

If the answer is affirmative, then the $L^{2}$-weak limit of $a_{k l}^{\varepsilon} \frac{\partial u^{\varepsilon}}{\partial x_{l}}$ is $M_{Y}\left(a_{k l}\right) \frac{\partial u^{*}}{\partial x_{l}}$ and therefore $\bar{A}$ is the homogenized operator. 
It is well-known that classical elliptic regularity results require that the coefficients be Lipschitz, i.e., $a_{k l} \in W_{\#}^{1, \infty}(Y)$, to obtain estimates in the space $H_{\mathrm{loc}}^{2}(\Omega)$. In our case, a direct application of these results yields

The above estimate is not uniform as $\varepsilon \rightarrow 0$.

$$
\left\|u^{\varepsilon}\right\|_{H_{\mathrm{loc}}^{2}(\Omega)} \leq C \varepsilon^{-1}\|f\|_{L_{\mathrm{loc}}^{2}(\Omega)} .
$$

Another idea is to prove the structure of the solution via multiple scales. In the book [8], we see an expansion of the form (with $y=x / \varepsilon$ )

$$
u^{\varepsilon}(x)=u^{*}(x)+\varepsilon\left\{\chi_{l}(y) \frac{\partial u^{*}}{\partial x_{l}}(x)+\widetilde{u}_{1}(x)\right\}+\varepsilon^{2}\left\{\chi_{l m}(y) \frac{\partial^{2} u^{*}}{\partial x_{l} \partial x_{m}}(x)+\chi_{l}(y) \frac{\partial \widetilde{u}_{1}}{\partial x_{l}}(x)+\widetilde{u}_{2}(x)\right\}+\cdots
$$

where $\chi_{l m}$ is the solution

$$
\left\{\begin{array}{l}
A \chi_{l m}=a_{l m}+a_{l k} \frac{\partial \chi_{m}}{\partial y_{k}}-\frac{\partial}{\partial y_{k}}\left(a_{k l} \chi_{m}\right)-q_{l m}, \text { in } \mathbb{R}^{N} \\
\chi_{l m} \in H_{\#}^{1}(Y), \quad M_{Y}\left(\chi_{l m}\right)=0
\end{array}\right.
$$

Of course, $u^{*}$ satisfies the homogenized equation $A^{*} u^{*}=f$ in $\Omega$. The above method also provides equations that must be satisfied by $\widetilde{u}_{1}, \widetilde{u}_{2}, \cdots$

where, for instance, when $j=1$, we have

$$
A^{*} \widetilde{u}_{j}=\widetilde{g}_{j} \quad \text { in } \Omega
$$

$$
\widetilde{g}_{1}(x)=b_{j k l} \frac{\partial^{3} u^{*}}{\partial x_{j} \partial x_{k} \partial x_{l}}(x)
$$

for some suitable constants.

Some comments on the above expansion are now in order. Because it contains infinitely many terms, it is not useful to establish $H_{\mathrm{loc}}^{2}$-estimates on $u^{\varepsilon}$. However, it offers an important insight into the difficulties in the offing. For instance, it shows that the second order derivatives of $\varepsilon$-term involve

$$
\varepsilon^{-1} \frac{\partial^{2} \chi_{l}}{\partial y_{j} \partial y_{k}}(y) \frac{\partial u^{*}}{\partial x_{l}}(x)
$$

Because of the presence of the negative power of $\varepsilon$, this term is not bounded as $\varepsilon \rightarrow 0$. An easy way out of the difficulty is to annihilate it by requiring that $\chi_{l}$ is a linear polynomial in $y$ which is equivalent to $\chi_{l}=0$. However, it is not at all clear whether this condition is going to be sufficient to overcome the difficulties posed by higher powers of $\varepsilon$. This is due to the following reasons:

(i) the second order derivatives of the coefficient of $\varepsilon^{2}$ contain terms involving derivatives of $u^{*}$ of higher order. We have no control on them with our hypothesis $f \in L_{\mathrm{loc}}^{2}(\Omega)$. More and more higher order derivatives of $u^{*}$ appear, and thus this difficulty is amplified when we go up in powers of $\varepsilon$;

(ii) there are also terms involving the second order derivatives of $\chi_{k l}$ and so it is natural to require that $\chi_{k l} \in W_{\#}^{2, \infty}(Y)$. It is classically known that such a regularity result on a cell problem is hard to come by if it is not impossible.

These fundamental issues and difficulties encountered in the physical space analysis may be the reason why $H^{2}$-regularity was not discussed in the literature. In the multiscale analysis, the operator $A^{\varepsilon}$ was straightaway restricted to functions of the form $v\left(x, \frac{x}{\varepsilon}\right)$ and this is not an optimal thing to do. Here, we use Fourier space analysis to prove a useful characterization for $H^{2}$-regularity. This demonstrates the power of the tools that we are using in the analysis. Given the above picture of difficulties, our results may be interpretated as follows: individually considered, above troublesome terms are not in $H_{\text {loc }}^{2}$; however, their sum is in $H_{\text {loc }}^{2}$ under the condition that $\chi_{l}=0 \forall l=1, \ldots, N$. 
The first step consists of localization, i.e., passage from $\Omega$ to $\mathbb{R}^{N}$. Since we are after all interested in local estimates, this is possible. Thus we consider $\left\{u^{\varepsilon}\right\}$ such that

$$
\left\{\begin{array}{l}
u^{\varepsilon} \rightarrow u^{*} \text { in } H^{1}\left(\mathbb{R}^{N}\right) \text { weak } \\
A^{\varepsilon} u^{\varepsilon}=f \quad \text { in } \quad \mathbb{R}^{N}
\end{array}\right.
$$

Theorem 1.9. Assume that $a_{k l} \in W_{\#}^{1, \infty}(Y)$. Then there is a constant $C$ independent of $\varepsilon$ such that

$$
\left|u^{\varepsilon}\right|_{H^{2}\left(\mathbb{R}^{N}\right)} \leq C\|f\|_{L^{2}\left(\mathbb{R}^{N}\right)}
$$

iff $\chi_{l}=0 \forall l$ or equivalently

$$
\frac{\partial a_{k l}}{\partial y_{k}}=0 \quad \forall l=1, \ldots, N
$$

By means of the homothecy $y=\frac{x}{\varepsilon}$, we can transform our problem to the equation $A u(y)=g(y)$ in $\mathbb{R}^{N}$. It has been our usual practice to analyze this equation in terms of Bloch decomposition of the operator $A$ described in Theorem 1.2. Unfortunately, this is useless in our present objective of estimating $\frac{\partial^{2} u}{\partial x_{k} \partial x_{l}}$ in $L^{2}\left(\mathbb{R}^{N}\right)$. This is because the Bloch decomposition is not invariant for the operator $\frac{\partial^{2}}{\partial x_{k} \partial x_{l}}$. This is a qualitative difference between homogeneous and inhomogeneous cases. (Recall, Fourier decomposition is invariant for all operators with constant coefficients.) To overcome this difficulty, our idea is to use the decomposition given in Theorem 1.3 which has the relevant invariance property. We see that our equation $A u=g$ in $\mathbb{R}^{N}$ can be equivalently decomposed into a family of parameterized problems in the cell $Y$ :

$$
\left\{\begin{array}{l}
A u_{\#}(u, \eta)=g_{\#}(y, \eta), \quad y \in \mathbb{R}^{N}, \\
u_{\#}(\cdot, \eta) \text { is }(\eta, Y)-\text { periodic. }
\end{array}\right.
$$

Yet another transform $u_{\#}(y, \eta)=\mathrm{e}^{i y \cdot \eta} U_{\#}(y, \eta)$ transforms the above problem to the following one with the usual periodic condition for the translated operator:

$$
\left\{\begin{array}{l}
A(\eta) U_{\#}=G_{\#} \text { in } \mathbb{R}^{N} \\
U_{\#} \text { is } Y-\text { periodic. }
\end{array}\right.
$$

Thus our problem is reduced to derive estimates on the second order derivatives of the above problem. We state the following result taken from [21].

Theorem 1.10. There exists a constant $C$ depending on $\|a\|_{W^{1, \infty}(Y)}$ but independent of $\eta \in Y^{\prime}$ such that

$$
|\eta|^{2}\left\|U_{\#}\right\|_{L^{2}(Y)}+|\eta|\left|U_{\#}\right|_{H^{1}(Y)}+\left|\eta\left\|\left.U_{\#}\right|_{H^{2}(Y)} \leq C\right\| G_{\#} \|_{L^{2}(Y)} .\right.
$$

This estimate degenerates as $\eta \rightarrow 0$. However, if $\frac{\partial a_{k l}}{\partial y_{k}}=0 \forall l$ then we have

$$
\left|U_{\#}\right|_{H^{2}(Y)} \leq C\left\|G_{\#}\right\|_{L^{2}(Y)}
$$

\section{ViBRATION PROBLEMS}

\subsection{Spectral asymptotic analysis}

As a second application of the Fourier approach, we show how it can be used to study the asymptotic behaviour of the spectrum of periodic structures in a bounded region $\Omega$ that we have been considering. As can be easily inferred, the physical motivation of such a study is to understand better wave propagation in periodic media, and it is well-known that this problem often relies on the analysis of the vibration modes of the medium. The wave equation is just a model; in truth, our original motivation comes from more complicated 
models describing the vibrations of fluid-solid structures. These physical models were proposed by Planchard in the early 80's (see e.g. $[19,40,41]$ ) and extensively studied using the Bloch-wave method in $[4,5,17,18,22]$.

To review part of these works, let us consider an arbitrary bounded domain $\Omega$ in $\mathbb{R}^{N}$ and, for each $\varepsilon>0$, let $A^{\varepsilon}$ be the operator considered in Chapter 1 and defined by

$$
A^{\varepsilon} \stackrel{\text { def }}{=}-\frac{\partial}{\partial x_{k}}\left(a_{k \ell}^{\varepsilon}(x) \frac{\partial}{\partial x_{\ell}}\right) \text { with } \quad a_{k \ell}^{\varepsilon}(x)=a_{k \ell}\left(\frac{x}{\varepsilon}\right) .
$$

Associated with $A^{\varepsilon}$, we consider the following spectral problem for the wave equation in $\Omega$ : find all couples $\left(\lambda^{\varepsilon}, v^{\varepsilon}\right) \in \mathbb{R} \times H_{0}^{1}(\Omega), v^{\varepsilon} \not \equiv 0$, such that

$$
A^{\varepsilon} v^{\varepsilon}=\lambda^{\varepsilon} v^{\varepsilon} \quad \text { in } \quad \Omega, \quad v^{\varepsilon} \in H_{0}^{1}(\Omega)
$$

At this point, we give a word of warning regarding the notation. In the articles just quoted, instead of working on the eigenvalue problem associated with $A^{\varepsilon}$, as we do here, work is done on the eigenvalues of $\left(A^{\varepsilon}\right)^{-1}$. The only consequence of this change of convention is that the eigen-variable $\lambda^{\varepsilon}$ in $(2.2)$ corresponds in these references to $\frac{1}{\lambda^{\varepsilon}}$, but of course, results in both cases are qualitatively equivalent.

With this in mind, let us denote by $\sigma^{\varepsilon}$ the set of eigenvalues of (2.2). As is well-known, $\sigma^{\varepsilon}$ is made of a countable sequence of eigenvalues which converge to $+\infty$ and each of them is of finite multiplicity, i.e.,

$$
\sigma^{\varepsilon}=\bigcup_{m \geq 1}\left\{\lambda_{m}^{\varepsilon}\right\} \quad \text { with } \quad 0<\lambda_{1}^{\varepsilon} \leq \cdots \leq \lambda_{m}^{\varepsilon} \leq \cdots \rightarrow+\infty
$$

To each $\lambda_{m}^{\varepsilon}$ is associated a normalized eigenfunction $v_{m}^{\varepsilon} \in L^{2}(\Omega)$ such that $\left\|v_{m}^{\varepsilon}\right\|_{L^{2}(\Omega)}=1$, and the family $\left\{v_{m}^{\varepsilon}\right\}_{m}$ is an orthonormal basis of $L^{2}(\Omega)$. It is further well-known that, as the period $\varepsilon$ tends to zero, $\sigma^{\varepsilon}$ converges to the spectrum $\sigma^{*}$ of the homogenized problem (see e.g. $\left.[10,31,39,48]\right)$ :

$$
A^{*} v=\lambda v \quad \text { in } \quad \Omega, \quad v \in H_{0}^{1}(\Omega),
$$

where $A^{*}$ is the homogenized operator. This is, for all $m \geq 1$,

$$
\lambda_{m}^{\varepsilon} \longrightarrow \lambda_{m}
$$

and the corresponding eigenfunction $v_{m}^{\varepsilon}$ converges strongly to $v_{m}$ in $L^{2}(\Omega)$, as $\varepsilon \rightarrow 0$. Here, $\lambda_{m}$ is the $m^{\text {th }}$ eigenvalue of the homogenized problem associated with $v_{m}$. Recall that $A^{*}$ is strictly elliptic and that therefore has a compact inverse in $L^{2}(\Omega)$. Its spectrum, denoted $\sigma^{*}$, is also made up of a countable sequence of eigenvalues of finite multiplicity.

Although it seems comprehensive, this convergence result does not completely describe the asymptotic behaviour of all the sequences of eigenvalues $\lambda^{\varepsilon}$ that converge to $+\infty$. In particular, letting $\varepsilon \rightarrow 0$ and $m \rightarrow+\infty$ it is possible to construct sequences of eigenvalues $\lambda_{m(\varepsilon)}^{\varepsilon}$ which converge to $+\infty$ and whose corresponding eigenfunctions do not converge strongly in $L^{2}(\Omega)$. In fact, they converge to 0 weakly in $L^{2}(\Omega)$. It is therefore interesting to consider suitable renormalizations of the set $\sigma^{\varepsilon}$ and study their asymptotic behaviour. More exactly, our goal in the remainder part of this section is to characterize the renormalized limits of the spectrum $\lim _{\varepsilon \rightarrow 0} \varepsilon^{\alpha} \sigma^{\varepsilon}$ where $\alpha$ is a positive number. This situation is called a low frequency limit, while the classical convergence result (2.4) and the strong convergence of the corresponding eigenvectors gives a high frequency limit (it is also called macroscopic limit).

Let us first consider the case of eigenvalues of the order of $\varepsilon^{-2}(\alpha=2)$ that, as we will see, corresponds to a critical case in the sense that the asymptotic behaviour of $\varepsilon^{2} \sigma^{\varepsilon}$ is special. First of all, let us begin by pinpointing the definition of this limit set: $\mathrm{By} \lim _{\varepsilon \rightarrow 0} \varepsilon^{2} \sigma^{\varepsilon}$ we mean the following set of cluster points:

$$
\lim _{\varepsilon \rightarrow 0} \varepsilon^{2} \sigma^{\varepsilon}=\left\{\begin{array}{l|l}
\lambda \in \mathbb{R}^{+} & \begin{array}{l}
\exists \text { a subsequence, still denoted by } \varepsilon \text { and } \exists\left(\lambda^{\varepsilon}, v^{\varepsilon}\right), \\
\text { solutions of }(2.2), \text { such that } \varepsilon^{2} \lambda^{\varepsilon} \longrightarrow \lambda
\end{array}
\end{array}\right\} .
$$


The main characterization result for this renormalized limit makes use of the Bloch spectrum, that we defined using the Bloch waves and the Bloch eigenvalues in Chapter 1 (see Sect. 1.5). To announce this result, we also

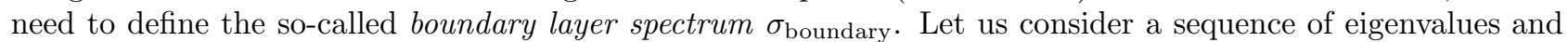
eigenvectors $\left(\lambda^{\varepsilon}, v^{\varepsilon}\right)$. If, for a subsequence still denoted by $\varepsilon$, there exists a limit $\lambda$ such that

$$
\left\{\begin{array}{l}
\left\|v^{\varepsilon}\right\|_{L^{2}(\Omega)}=1 \quad \text { and } \quad \lim _{\varepsilon \rightarrow 0} \varepsilon^{2} \lambda^{\varepsilon}=\lambda \\
\lim _{\varepsilon \rightarrow 0}\left\|v^{\varepsilon}\right\|_{L^{2}(\omega)}=0
\end{array}\right.
$$

for any subset $\omega$ with $\bar{\omega} \subset \Omega$, then $\lambda$ is said to belong to $\sigma_{\text {boundary }}$.

From a physical point of view, $\sigma_{\text {boundary }}$ corresponds to sequences of eigenvectors concentrating near the boundary of $\Omega$. Comparing its definition with that of the macroscopic spectrum $\sigma_{\mathrm{Bloch}}$, we see that $\sigma_{\text {boundary }}$ may depend on the choice of the sequence $\varepsilon$. Our main result is

\section{Theorem 2.1.}

$$
\lim _{\varepsilon \rightarrow 0} \varepsilon^{2} \sigma^{\varepsilon}=\sigma_{\text {boundary }} \cup \sigma_{\text {Bloch }}
$$

The proof of the above result is sketched in the next section. It relies on the notion of Bloch limiting measures which play, more or less, the role of semi-classical (or Wigner) measures in the context of Schrödinger equation (see e.g. $[27,29,32,33,46]$ ). Here, as already mentioned, the scaling $\varepsilon^{-2}$ of the eigenvalues can be interpreted as a critical size. Indeed, for any other scaling, we find a simpler result since there is no interaction between the period size $\varepsilon$ and the frequency size:

Theorem 2.2. Let $a_{\varepsilon} \in \mathbb{R}^{+}$be a sequence converging to 0 with $\varepsilon$ and such that, either

$$
\lim _{\varepsilon \rightarrow 0} \frac{a_{\varepsilon}}{\varepsilon}=0, \quad \text { or } \quad \lim _{\varepsilon \rightarrow 0} \frac{a_{\varepsilon}}{\varepsilon}=+\infty .
$$

Then,

$$
\lim _{\varepsilon \rightarrow 0}\left(a_{\varepsilon}\right)^{2} \sigma_{\varepsilon}=\mathbb{R}^{+}
$$

The above theorem is consistent with Weyl's asymptotic distribution of eigenvalues for the Laplacian. Indeed, if there were no periodic heterogeneities (i.e., if the coefficients $a_{k \ell}(y)$ were constants), then Weyl's result would imply that the renormalized limit of the spectrum is always the entire positive half line. The reader can find a proof of Theorem 2.2 in [3].

Theorem 2.1 motivates the important question of how to characterize explicitly the boundary layer spectrum. Indeed, the definition of $\sigma_{\text {boundary }}$ given above is not very enlightening, because it does not characterize it as the spectrum of an operator associated with the boundary $\partial \Omega$ of $\Omega$. In particular, it is not clear whether $\sigma_{\text {boundary }}$

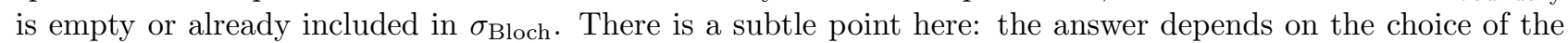
sequence $\varepsilon$. A striking result has been obtained in [11] when the sequence $\varepsilon$ takes all real values close to 0 .

Theorem 2.3. Let $\varepsilon$ be the sequence of all real numbers in the interval $\left(0, \varepsilon_{0}\right)$ with $\varepsilon_{0}>0$. Then

$$
\lim _{\varepsilon \rightarrow 0} \varepsilon^{2} \sigma_{\varepsilon}=\mathbb{R}^{+}
$$

which means that the boundary layer spectrum $\sigma_{\text {boundary }}$ must necessarily fill the gaps of the Bloch spectrum $\sigma_{\text {Bloch }}$.

In Theorem 2.3 it is crucial that the sequence $\varepsilon$ takes all possible values near 0 . For a general domain $\Omega$ and a

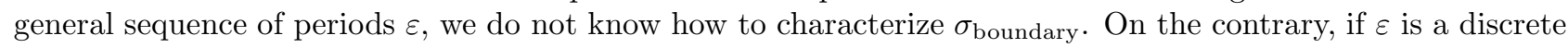
sequence and $\Omega$ has a piecewise flat boundary, we obtain a complete characterization of $\sigma_{\text {boundary }}$ which may 


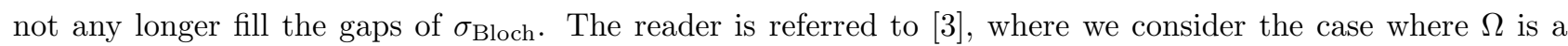
rectangle with integer dimensions and the sequence $\varepsilon$ is given exactly by $\varepsilon_{n}=1 / n, n \in \mathbb{N}^{*}$, which means that $\Omega$ is always a union of periodic cells of size $\varepsilon_{n}$. Suitable generalizations of the method presented below lead in this

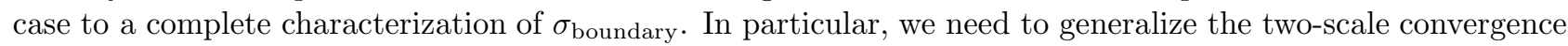
for treating the case of boundary layers (see [4]).

\subsection{Asymptotics of the spectrum}

This section is devoted to the proof of Theorem 2.1. For a better organization of the exposition, we shall divide the proof into two main steps.

\subsubsection{Bloch wave homogenization}

Studying a specific spectral problem which arises in fluid-solid interactions, Allaire and Conca introduced in [5] a new method of homogenization, the so-called Bloch-wave homogenization method (which is a mix of two-scale convergence and Bloch-wave decomposition), which has been very useful for tackling the asymptotic behaviour of the spectrum of periodic structures. By means of this method, we prove in this paragraph that

$$
\sigma_{\text {Bloch }} \subset \sigma^{\infty}
$$

where $\sigma^{\infty}$ is used as a shorthand of $\lim _{\varepsilon \rightarrow 0} \varepsilon^{2} \sigma^{\varepsilon}$. To this end, let us rewrite the spectral problem (2.2) as follows: find $\left(\mu^{\varepsilon}, v^{\varepsilon}\right), v^{\varepsilon} \not \equiv 0$, such that

$$
\varepsilon^{2} A^{\varepsilon} v^{\varepsilon}+v^{\varepsilon}=\frac{1}{\mu^{\varepsilon}} v^{\varepsilon} \quad \text { in } \quad \Omega, \quad v^{\varepsilon} \in H_{0}^{1}(\Omega) .
$$

Observe that this passage leaves the eigenfunctions unchanged and changes the eigenvalues $\lambda_{m}^{\varepsilon}$ to $\mu_{m}^{\varepsilon}=1 /(1+$ $\varepsilon^{2} \lambda_{m}^{\varepsilon}$ ) and hence, $\mu^{\varepsilon} \sim 1$ iff $\lambda^{\varepsilon} \sim \varepsilon^{-2}$.

To resolve the above problem, it is a classical technique to introduce the so-called Green's operator $S^{\varepsilon} \in \mathcal{L}\left(L^{2}(\Omega)\right)$ which is defined as $S^{\varepsilon} f=u^{\varepsilon}$, where $u^{\varepsilon}$ is the unique solution of

$$
\varepsilon^{2} A^{\varepsilon} u^{\varepsilon}+u^{\varepsilon}=f \quad \text { in } \quad \Omega, \quad u^{\varepsilon} \in H_{0}^{1}(\Omega) .
$$

The starting point in order to characterize $\sigma^{\infty}$ is to pass to the limit in the above the problem. Well now, it is an easy exercise in perturbation's theory to show that $u^{\varepsilon}$ converges weakly in $L^{2}(\Omega)$ to $f$. This implies that $S^{\varepsilon}$ converges weakly to the identity operator, a property which does not yield any information on the asymptotic behaviour of its spectrum. To overcome this difficulty, our strategy is to obtain a strong convergence and the main idea in obtaining this is to extend the operator $S^{\varepsilon}$ by embedding $L^{2}(\Omega)$ into a larger space of two-scale functions. Since the lack of strong convergence is due to oscillations, the enlarged space must incorporate them. Of course, the extension of $S^{\varepsilon}$ must be done in such a way as to essentially maintain the same spectrum as $S^{\varepsilon}$. With this in mind, for any positive integer $K \geq 1$, denoting by $K Y$ the cube $[0, K]^{N}$, we define an extension operator $S_{K}^{\varepsilon} \in \mathcal{L}\left(L^{2}\left(\Omega ; L_{\#}^{2}(K Y)\right)\right)$ by $S_{K}^{\varepsilon}=E_{K}^{\varepsilon} S^{\varepsilon} P_{K}^{\varepsilon}$, where $P_{K}^{\varepsilon}$ and $E_{K}^{\varepsilon}$ are respectively a projection from $L^{2}\left(\Omega ; L_{\#}^{2}(K Y)\right)$ into $L^{2}(\Omega)$ and an extension from $L^{2}(\Omega)$ into $L^{2}\left(\Omega ; L_{\#}^{2}(K Y)\right)$. To insure that $S_{K}^{\varepsilon}$ is still selfadjoint, we ask $P_{K}^{\varepsilon}$ and $E_{K}^{\varepsilon}$ to be adjoint of one another. To ensure that $S^{\varepsilon}$ and $S_{K}^{\varepsilon}$ have the same spectrum, denoted by $\tilde{\sigma}^{\varepsilon}$, we ask the product $P_{K}^{\varepsilon} E_{K}^{\varepsilon}$ to be equal to the identity in $L^{2}(\Omega)$. Such conditions are satisfied by

$$
\left\{\begin{array}{l}
\forall \varphi \in L^{2}\left(\Omega ; L_{\#}^{2}(K Y)\right), \quad\left(P_{K}^{\varepsilon} \varphi\right)(x)=\sum_{i=1}^{n(\varepsilon)} \chi_{i}^{\varepsilon}(x) \frac{1}{(K \varepsilon)^{N}} \int_{Y_{i}^{\varepsilon}} \varphi\left(x^{\prime}, \frac{x}{\varepsilon}\right) \mathrm{d} x^{\prime} \\
\forall f \in L^{2}(\Omega), \quad\left(E_{K}^{\varepsilon} f\right)(x, y)=\sum_{i=1}^{n(\varepsilon)} \chi_{i}^{\varepsilon}(x) f\left(x_{i}^{\varepsilon}+\varepsilon y\right),
\end{array}\right.
$$


where the family $\left(Y_{i}^{\varepsilon}\right)_{1 \leq i \leq n(\varepsilon)}$ of non-overlapping cells of the type $[0 ; K \varepsilon]^{N}$ covers $\Omega\left(\chi_{i}^{\varepsilon}\right.$ is the characteristic function of $Y_{i}^{\varepsilon}$ and $x_{i}^{\varepsilon}$ its origin).

Proposition 2.4. The sequence $S_{K}^{\varepsilon}$ converges strongly to a limit operator $S_{K}$ in the sense that, for any $\varphi(x, y) \in L^{2}\left(\Omega ; L_{\#}^{2}(K Y)\right), S_{K}^{\varepsilon} \varphi$ converges strongly to $S_{K} \varphi$ in $L^{2}\left(\Omega ; L_{\#}^{2}(K Y)\right)$ and $S_{K} \varphi=u_{K}$ is the unique solution in $L^{2}\left(\Omega ; H_{\#}^{1}(K Y)\right)$ of

$$
A u_{K}+u_{K}=\varphi \text { in } \Omega \times K Y \text {. }
$$

Moreover, $S_{K}$ is a self-adjoint non-compact operator in $L^{2}\left(\Omega ; L_{\#}^{2}(K Y)\right)$.

The convergence of $S_{K}^{\varepsilon}$ to $S_{K}$ cannot be uniform since $S_{K}^{\varepsilon}$ is compact, but not $S_{K}$. From the strong convergence of operators, we deduce the lower semi-continuity of the spectrum, i.e.,

Corollary 2.5. Let $\sigma_{K}$ denote the spectrum of $S_{K}$. Since $S_{K}^{\varepsilon}$ converges strongly to $S_{K}$, we have

$$
\sigma_{K} \subset \lim _{\varepsilon \rightarrow 0} \tilde{\sigma}_{\varepsilon} .
$$

The key ingredient in the proof of Proposition 2.4 is the notion of two-scale convergence introduced in [2,37], that we briefly recall in the sequel.

Lemma 2.6. Let $u^{\varepsilon}$ be a bounded sequence in $L^{2}(\Omega)$. Then there exists a subsequence, which we still denote by $\varepsilon$, and a limit function $u^{0}(x, y) \in L^{2}(\Omega \times Y)$ such that

$$
\lim _{\varepsilon \rightarrow 0} \int_{\Omega} u^{\varepsilon}(x) \varphi\left(x, \frac{x}{\varepsilon}\right) \mathrm{d} x=\frac{1}{|Y|} \int_{\Omega} \int_{Y} u^{0}(x, y) \varphi(x, y) \mathrm{d} x \mathrm{~d} y
$$

for all functions $\varphi(x, y) \in L^{2}\left(\Omega ; \mathcal{C}_{\#}^{0}(Y)\right)$.

Proof of Proposition 2.4. Let $\psi^{\varepsilon}(x, y)$ be a sequence converging weakly to $\psi(x, y)$ in the space $L^{2}\left(\Omega ; L_{\#}^{2}(K Y)\right)$. For any $\varphi \in L^{2}\left(\Omega ; L_{\#}^{2}(K Y)\right)$, we need to show that

$$
\lim _{\varepsilon \rightarrow 0} \int_{\Omega} \int_{K Y}\left(S_{K}^{\varepsilon} \varphi\right) \psi^{\varepsilon} \mathrm{d} x \mathrm{~d} y=\int_{\Omega} \int_{K Y}\left(S_{K} \varphi\right) \psi \mathrm{d} x \mathrm{~d} y .
$$

By definition of $S_{K}^{\varepsilon}$, one has

$$
\frac{1}{K^{N}} \int_{\Omega} \int_{K Y}\left(S_{K}^{\varepsilon} \varphi\right) \psi^{\varepsilon} \mathrm{d} x \mathrm{~d} y=\int_{\Omega}\left(S^{\varepsilon} P_{K}^{\varepsilon} \varphi\right)\left(E_{K}^{\varepsilon}\right)^{*} \psi^{\varepsilon} \mathrm{d} x=\int_{\Omega} u^{\varepsilon}\left(P_{K}^{\varepsilon} \psi^{\varepsilon}\right) \mathrm{d} x,
$$

where $u^{\varepsilon}$ is the solution of (2.8) with right hand side $f=P_{K}^{\varepsilon} \varphi$. Using Lemma 2.6 one can show that $u^{\varepsilon}$ two-scale converges "strongly" to the solution $u_{K}$ of $(2.9)$, while the sequence $P_{K}^{\varepsilon} \psi^{\varepsilon}$ two-scale converges "weakly" to $\psi$. Then, passing to the limit in (2.10) yields

$$
\frac{1}{K^{N}} \int_{\Omega} \int_{K Y} u_{K} \psi \mathrm{d} x \mathrm{~d} y=\frac{1}{K^{N}} \int_{\Omega} \int_{K Y}\left(S_{K} \varphi\right) \psi \mathrm{d} x \mathrm{~d} y,
$$

which concludes the proof (see [5] for a more detailed proof in a similar case).

To compute the spectrum $\sigma_{K}$, we use a discrete Bloch-wave decomposition in $L_{\#}^{2}(K Y)$ (see [1]). This allows us to diagonalize $S_{K}$. 
Lemma 2.7 [1]. For any function $\varphi(y) \in L_{\#}^{2}(K Y)$ there exists a unique family $\left\{\varphi_{j}(y)\right\} \in L_{\#}^{2}(Y)^{K^{N}}$, indexed by a multi-index $j$ whose $N$ components belong to $\{0, \ldots, K-1\}$, such that the following inverse formula and Parseval's equality hold:

$$
\begin{aligned}
\varphi(y) & =\sum_{0 \leq j \leq K-1} \varphi_{j}(y) \mathrm{e}^{i \frac{j \cdot y}{K}}, \\
\frac{1}{K^{N}} \int_{K Y}|\varphi|^{2} \mathrm{~d} y & =\sum_{0 \leq j \leq K-1} \int_{Y}\left|\varphi_{j}\right|^{2} \mathrm{~d} y .
\end{aligned}
$$

This decomposition defines a unitary isometry, denoted by $B$, from $L_{\#}^{2}(K Y)$ into $L_{\#}^{2}(Y)^{K^{N}}$.

From the above lemma, we easily deduce the following:

Proposition 2.8. The operator $S_{K}$ can be diagonalized as

$$
S_{K}=B^{*} T_{K} B \quad \text { with } \quad T_{K}=\operatorname{diag}\left[\left(T_{j / K}\right)_{0 \leq j \leq K-1}\right]
$$

where, for each Bloch frequency $\eta=j / K, T_{\eta}$ is defined as an element of $\mathcal{L}\left(L^{2}\left(\Omega ; L_{\#}^{2}(Y)\right)\right)$ as $T_{\eta} \varphi=u^{0}$, where $u^{0}=u^{0}(x, y)$ is the unique solution in $L^{2}\left(\Omega ; H_{\#}^{1}(Y)\right)$ of

$$
A(\eta) u^{0}+u^{0}=\mathrm{e}^{i y \cdot \eta} \varphi \quad \text { in } \quad \Omega \times Y
$$

We recognize in the operators $T_{\eta}$ a simple transformation of the Green's operators associated with the shifted operator. To conclude the proof of (2.6), it suffices to remark that, as $K$ goes to infinity, the discrete set of the Bloch frequencies $j / K$ becomes dense in $Y^{\prime}$ (see [3] for more details).

\subsubsection{Completeness of the spectrum}

We now address the question of whether the Bloch spectrum is enough to completely characterize the limit set $\sigma^{\infty}$. In other words, we seek for what we call a result of completeness. Well now, it turns out in this case that the Bloch spectrum is usually not enough to describe $\sigma^{\infty}$ because there is another source of limiting spectrum which is not taken into account in our analysis. This source corresponds to sequences of eigenvectors of (2.2) concentrating near the boundary $\partial \Omega$ of $\Omega$. They behave as boundary layers in the sense that they converge strongly to zero inside the domain. It is therefore clear that the oscillations of these sequences of eigenvectors cannot be captured by the usual homogenization method, neither are they captured in $\sigma_{\text {Bloch }}$ since the Bloch waves are insensitive to the boundary.

Following this line of reasoning, we prove in this paragraph that

$$
\sigma^{\infty}=\lim _{\varepsilon \rightarrow 0} \varepsilon^{2} \sigma^{\varepsilon} \subset \sigma_{\text {boundary }} \cup \sigma_{\text {Bloch }} .
$$

To this end, we consider any sequence $\left(\lambda^{\varepsilon}, v^{\varepsilon}\right) \in \mathbb{R}^{+} \times H_{0}^{1}(\Omega)$, solutions of the spectral problem (2.2), such that (up to a subsequence) there exists a limit $\lambda$ satisfying

$$
\left\|v^{\varepsilon}\right\|_{L^{2}(\Omega)}=1 \text { and } \quad \lim _{\varepsilon \rightarrow 0} \varepsilon^{2} \lambda^{\varepsilon}=\lambda
$$

If, for any subset $\omega$ such that $\bar{\omega} \subset \Omega$,

$$
\lim _{\varepsilon \rightarrow 0}\left\|v^{\varepsilon}\right\|_{L^{2}(\omega)}=0
$$

then, by definition, the limit eigenvalue $\lambda$ belongs to the boundary layer spectrum. Therefore, to complete the proof of Theorem 2.1, it remains to prove that, if there exists a subset $\omega$ and a subsequence, still denoted by $\varepsilon$, 
such that

$$
\lim _{\varepsilon \rightarrow 0}\left\|v^{\varepsilon}\right\|_{L^{2}(\omega)}=c>0
$$

then $\lambda$ belongs to the Bloch spectrum.

Now, if the above assumption (2.14) is fulfilled, then there exists a smooth cut-off function $\varphi \in \mathcal{D}(\Omega)$ such that $\varphi \equiv 1$ in $\omega$, and defining a sequence

$$
u^{\varepsilon}=\frac{\varphi v^{\varepsilon}}{\left\|\varphi v^{\varepsilon}\right\|_{L^{2}(\Omega)}},
$$

it is easily seen that $u^{\varepsilon}$ is a sequence of quasi-eigenvectors in the sense that it has compact support in $\bar{\Omega}$, $\left\|u^{\varepsilon}\right\|_{L^{2}\left(\mathbb{R}^{N}\right)}=1$, and it satisfies

$$
A^{\varepsilon} u^{\varepsilon}=\lambda^{\varepsilon} u^{\varepsilon}+r^{\varepsilon} \quad \text { in } \quad \mathbb{R}^{N}
$$

where $r^{\varepsilon} \in L^{2}\left(\mathbb{R}^{N}\right)$ is a negligible remainder term satisfying $\lim _{\varepsilon \rightarrow 0}\left\langle r^{\varepsilon}, w^{\varepsilon}\right\rangle_{H^{-1}(\Omega), H_{0}^{1}(\Omega)}=0$, for any sequence $w^{\varepsilon}$ such that $\left\|w^{\varepsilon}\right\|_{L^{2}(\Omega)}+\varepsilon\left\|\nabla w^{\varepsilon}\right\|_{L^{2}(\Omega)^{N}} \leq C$.

The above equation (2.15) in terms of Bloch transforms reduces to

$$
\lambda_{m}(\eta) B_{m}^{\varepsilon} u^{\varepsilon}(\eta)=\varepsilon^{2} \lambda^{\varepsilon} B_{m}^{\varepsilon} u^{\varepsilon}(\eta)+\varepsilon^{2} B_{m}^{\varepsilon} r_{m}^{\varepsilon} \quad \text { in } \quad Y^{\prime},
$$

for all $m \geq 1$, where $B_{m}^{\varepsilon} u^{\varepsilon}$ is the $m^{t h}$ Bloch coefficient of $u^{\varepsilon}$. Let us multiply these equations by $\psi_{m}(\eta) \overline{B_{m}^{\varepsilon} u^{\varepsilon}(\eta)}$, where $\psi_{m}(\eta)$ is a given continuous function in $\mathcal{C}_{\#}^{0}\left(\overline{Y^{\prime}}\right)$. Adding up on $m$ and integrating in $\eta$, thanks to Parseval's equality, we obtain the following key relation

$$
\sum_{m \geq 1} \int_{Y^{\prime}} \psi_{m}(\eta)\left(\lambda_{m}(\eta)-\varepsilon^{2} \lambda^{\varepsilon}\right)\left|B_{m}^{\varepsilon} u^{\varepsilon}(\eta)\right|^{2} \mathrm{~d} \eta=O(\varepsilon)
$$

where $O(\varepsilon)$ tends to zero with $\varepsilon$.

For each $m \geq 1$, we associate to $u^{\varepsilon}$ a function $h_{m}^{\varepsilon}$ defined for $\eta \in Y^{\prime}$ by $h_{m}^{\varepsilon}(\eta)=\left|B_{m}^{\varepsilon} u^{\varepsilon}(\eta)\right|^{2}$. Since $u^{\varepsilon}$ has a unit norm in $L^{2}\left(\mathbb{R}^{N}\right)$, by Parseval's equality we have

$$
\sum_{m \geq 1}\left\|h_{m}^{\varepsilon}\right\|_{L^{1}\left(Y^{\prime}\right)}=\sum_{m \geq 1} \int_{Y^{\prime}}\left|B_{m}^{\varepsilon} u^{\varepsilon}(\eta)\right|^{2} \mathrm{~d} \eta=\left\|u^{\varepsilon}\right\|_{L^{2}\left(\mathbb{R}^{N}\right)}^{2}=1 .
$$

Each $h_{m}^{\varepsilon}$ is therefore bounded in $L^{1}\left(Y^{\prime}\right)$ and the sum of their norms is equal to 1 . Up to a subsequence, there exists a family of limit Radon measures $\left\{\nu_{m}(\eta)\right\}_{m \geq 1}$ such that each $h_{m}^{\varepsilon}$ converges to $\nu_{m}$ in the sense of vague convergence of measures. Of course, the limit measures are all non-negative, but they may well be zero. We can call these Bloch-limiting measures. Let us show that they satisfy

$$
\sum_{m \geq 1} \int_{Y^{\prime}} \mathrm{d} \nu_{m}(\eta)=1,
$$

which would prove that at least one of them is not identically zero. To prove (2.17), we use the following discrete version of the classical dominated convergence theorem. 
Lemma 2.9. For each $\varepsilon>0$, let $\left\{a_{m}^{\varepsilon}\right\}_{m}$ be a sequence of real numbers such that the series $\sum_{m \geq 1} a_{m}^{\varepsilon}$ is convergent. Assume that the following conditions hold:

$$
\begin{aligned}
& \text { (i) } \forall m \geq 1, \exists a_{m} \in \mathbb{R}^{N} ; a_{m}^{\varepsilon} \longrightarrow a_{m} \text {, as } \varepsilon \rightarrow 0, \\
& \text { (ii) }\left\{\begin{array}{l}
\exists \text { a convergent series } \sum_{m \geq 1} \zeta_{m} ;\left|a_{m}^{\varepsilon}\right| \leq \zeta_{m} \quad \forall m \text {, or } \\
\forall \delta>0, \exists m_{\delta} \geq 1 ; \sum_{m \geq m_{\delta}} a_{m}^{\varepsilon} \leq \delta \quad \forall \varepsilon .
\end{array}\right.
\end{aligned}
$$

Then the series $\sum_{m \geq 1} a_{m}^{\varepsilon}$ converges as $\varepsilon$ goes to zero and its limit is $\sum_{m \geq 1} a_{m}$.

Let us apply this lemma to the sequence $a_{m}^{\varepsilon}=\int_{Y^{\prime}} h_{m}^{\varepsilon}(\eta) \mathrm{d} \eta$. First, for each fixed $m$ we have

$$
\lim _{\varepsilon \rightarrow 0} \int_{Y^{\prime}} h_{m}^{\varepsilon}(\eta) \mathrm{d} \eta=\int_{Y^{\prime}} \mathrm{d} \nu_{m}(\eta)
$$

Next, let us assume that the second condition in (ii) is not satisfied. Then there exists $\delta>0$, a subsequence, still denoted by $\varepsilon$, and a sequence of integers $m(\varepsilon)$, going to $+\infty$, such that

$$
\sum_{m \geq m(\varepsilon)_{Y^{\prime}}} \int_{m}^{\varepsilon} h_{m}^{\varepsilon}(\eta) \mathrm{d} \eta>\delta .
$$

Thanks to the boundedness of $\varepsilon \nabla u^{\varepsilon}$, by Parseval's Identity we have

$$
M \geq \varepsilon^{2}\left\|\nabla u_{\varepsilon}\right\|_{L^{2}\left(\mathbb{R}^{N}\right)}^{2} \geq \frac{1}{\beta} \sum_{m \geq 1_{Y^{\prime}}} \int_{m}(\eta) h_{m}^{\varepsilon}(\eta) \mathrm{d} \eta \geq \frac{\delta}{\beta} \min _{\eta \in Y^{\prime}} \lambda_{m(\varepsilon)}(\eta),
$$

where $\beta=\max _{k, \ell}\left\|a_{k, \ell}\right\|_{L^{\infty}(Y)}$. But, this is a contradiction, since for any $\eta \in Y^{\prime} \lim _{m \rightarrow+\infty} \lambda_{m}(\eta)=+\infty$. This completes the proof of $(2.17)$.

Since the test function $\psi$ and the Bloch eigenvalues $\lambda_{m}$ are continuous in $\eta$, again with the help of Lemma 2.9 it is easily seen that one can pass to the limit in the relation $(2.16)$ :

$$
\sum_{m \geq 1} \int_{Y^{\prime}} \psi_{m}(\eta)\left(\lambda_{m}(\eta)-\lambda\right) \mathrm{d} \nu_{m}(\eta)=0
$$

Since at least one of the Bloch limiting measures $\nu_{m}$ is not trivial, there exists an energy level $m \geq 1$ and a Bloch frequency $\eta$ such that

which finishes the proof of Theorem 2.1.

$$
\lambda=\lambda_{m}(\eta)
$$

\section{REFERENCES}

[1] F. Aguirre and C. Conca, Eigenfrequencies of a tube bundle immersed in a fluid. Appl. Math. Optim. 18 (1988) 1-38.

[2] G. Allaire, Homogenization and two-scale convergence. SIAM J. Math. Anal. 23 (1992) 1482-1518.

[3] G. Allaire and C. Conca, Bloch-wave homogenization and spectral asymptotic analysis. J. Math. Pures Appl. 77 (1998) 153-208.

[4] G. Allaire and C. Conca, Boundary layers in the homogenization of a spectral problem in fluid-solid structures. SIAM J. Math. Anal. 29 (1997) 343-379.

[5] G. Allaire and C. Conca, Bloch wave homogenization for a spectral problem in fluid-solid structures. Arch. Rational Mech. Anal. 135 (1996) 197-257. 
[6] G. Allaire and C. Conca, Analyse asymptotique spectrale de l'équation des ondes. Homogénéisation par ondes de Bloch. $C . R$. Acad. Sci. Paris Sér. I Math. 321 (1995) 293-298.

[7] G. Allaire and C. Conca, Analyse asymptotique spectrale de l'équation des ondes. Complétude du spectre de Bloch. $C . R$. Acad. Sci. Paris Sér. I Math. 321 (1995) 557-562.

[8] A. Bensoussan, J.-L. Lions and G. Papanicolaou, Asymptotic Analysis in Periodic Structures. North-Holland, Amsterdam (1978).

[9] F. Bloch, Über die Quantenmechanik der Electronen in Kristallgittern. Z. Phys. 52 (1928) 555-600.

[10] L. Boccardo and P. Marcellini, Sulla convergenza delle soluzioni di disequazioni variazionali. Ann. Mat. Pura Appl. 4 (1977) 137-159.

[11] C. Castro and E. Zuazua, Une remarque sur l'analyse asymptotique spectrale en homogénéisation. C. R. Acad. Sci. Paris Sér. I Math. 322 (1996) 1043-1048.

[12] A. Cherkaev and R. Kohn, Topics in the Mathematical Modelling of Composite Materials. Birkhäuser, Boston (1997).

[13] C. Conca, S. Natesan and M. Vanninathan, Numerical experiments with the Bloch-Floquet approach in homogenization (to appear).

[14] C. Conca, R. Orive and M. Vanninathan, Bloch Approximation in Homogenization and Applications. SIAM J. Math. Anal. (in press).

[15] C. Conca, R. Orive and M. Vanninathan, Bloch Approximation in bounded domains. Preprint (2002).

[16] C. Conca, R. Orive and M. Vanninathan, Application of Bloch decomposition in wave propagation problems (in preparation).

[17] C. Conca, J. Planchard and M. Vanninathan, Fluids and Periodic Structures. J. Wiley and Sons/Masson, New York/Paris, Collection RAM 38 (1995).

[18] C. Conca, J. Planchard and M. Vanninathan, Limiting behaviour of a spectral problem in fluid-solid structures. Asymp. Anal. 6 (1993) 365-389.

[19] C. Conca, J. Planchard, B. Thomas and M. Vanninathan, Problèmes Mathématiques en Couplage Fluide-Structure. Applications aux Faisceaux Tubulaires. Eyrolles, Paris (1994).

[20] C. Conca and M. Vanninathan, Homogenization of periodic structures via Bloch decomposition. SIAM J. Appl. Math. 57 (1997) 1639-1659.

[21] C. Conca and M. Vanninathan, On uniform $H^{2}$-estimates in periodic homogenization. Proc. Roy. Soc. Edinburgh Sect. A 131 (2001) 499-517.

[22] C. Conca and M. Vanninathan, A spectral problem arising in fluid-solid structures. Comput. Methods Appl. Mech. Engrg. 69 (1988) 215-242.

[23] G. Dal Maso, An Introduction to $\Gamma$-Convergence. Birkhäuser, Boston (1993).

[24] A. Figotin and P. Kuchment, Band-gap structure of spectra of periodic dielectric and accoustic media. I, scalar model. SIAM J. Appl. Math. 56 (1996) 68-88.

[25] G. Floquet, Sur les équations différentielles linéaires à coefficients périodiques. Ann. École Norm. Sér. 212 (1883) 47-89.

[26] I.M. Gelfand, Expansion in series of eigenfunctions of an equation with periodic coefficients. Dokl. Akad. Nauk SSSR 73 (1950) $1117-1120$.

[27] P. Gérard, Mesures semi-classiques et ondes de Bloch, in Séminaire Equations aux Dérivées Partielles, Vol. 16, $1990-1991$. École Polytechnique, Palaiseau (1991).

[28] P. Gérard, Microlocal defect measures. Comm. Partial Differential Equation 16 (1991) 1761-1794.

[29] P. Gérard, P.A. Markowich, N.J. Mauser and F. Poupaud, Homogenization limits and Wigner transforms. Comm. Pure. Appl. Math. 50 (1997) 321-377.

[30] L. Hörmander, Analysis of Linear Partial Differential Operators III. Springer-Verlag, Berlin (1985).

[31] S. Kesavan, Homogenization of elliptic eigenvalue problems, I and II. Appl. Math. Optim. 5 (1979) 153-167, 197-216.

[32] P.L. Lions and T. Paul, Sur les mesures de Wigner. Revista Math. Iberoamer. 9 (1993) 553-618.

[33] P.A. Markowich, N.J. Mauser and F. Poupaud, A Wigner function approach to semiclassical limits: electrons in a periodic potential. J. Math. Phys. 35 (1994) 1066-1094.

[34] R. Morgan and I. Babuška, An approach for constructing families of homogenized equations for periodic media I and II. SIAM J. Math. Anal. 2 (1991) 1-15, 16-33.

[35] F. Murat, (1977-78) H-Convergence, Séminaire d'Analyse Fonctionnelle et Numérique de l'Université d'Alger, mimeographed notes. English translation: Murat and L. Tartar, H-Convergence, in F. Topics in the Mathematical Modelling of Composite Materials, edited by A. Cherkaev and R. Kohn. Birkhäuser Verlag, Boston. Series Progress in Nonlinear Differential Equations and their Applications 31 (1977).

[36] F. Murat, A survey on compensated compactness, in Contributions to Modern Calculus of Variations, edited by L. Cesari, Pitman Res. Notes in Math. Ser. 148 (1987) 145-183.

[37] G. Nguetseng, A general convergence result for a functional related to the theory of homogenization. SIAM J. Math. Anal. 20 (1989) 608-623.

[38] F. Odeh and J.B. Keller, Partial differential equations with periodic coefficients and Bloch waves in crystals. J. Math. Phys. 5 (1964) 1499-1504. 
[39] O.A. Oleinik, A.S. Shamaev and G.A. Yosifian, On the limiting behaviour of a sequence of operators defined in different Hilbert's spaces. Upsekhi Math. Nauk. 44 (1989) 157-158.

[40] J. Planchard, Global behaviour of large elastic tube-bundles immersed in a fluid. Comput. Mech. 2 (1987) 105-118.

[41] J. Planchard, Eigenfrequencies of a tube-bundle placed in a confined fluid. Comput. Methods Appl. Mech. Engrg. 30 (1982) 75-93.

[42] M. Reed and B. Simon, Methods of Modern Mathematical Physics. I. Functional Analysis, II. Fourier Analysis and SelfAdjointness, III. Scattering Theory, IV. Analysis of Operators. Academic Press, New York (1972-78).

[43] E. Sánchez-Palencia, Non-Homogeneous Media and Vibration Theory. Springer-Verlag, Berlin. Lecture Notes in Phys. 127 (1980)

[44] J. Sánchez-Hubert and E. Sánchez-Palencia, Vibration and Coupling of Continuous Systems. Asymptotic Methods. SpringerVerlag, Berlin (1989)

[45] F. Santosa and W.W. Symes, A dispersive effective medium for wave propagation in periodic composites. SIAM J. Appl. Math. 51 (1991) 984-1005.

[46] L. Tartar, $H$-measures, a new approach for studying homogenization, oscillations and concentration effects in partial differential equations. Proc. Roy. Soc. Edinburgh Sect. A 115 (1990) 193-230.

[47] L. Tartar, Problèmes d'Homogénéisation dans les Equations aux Dérivées Partielles, Cours Peccot au Collège de France (1977). Partially written in F. Murat [25].

[48] M. Vanninathan, Homogenization and eigenvalue problems in perforated domains. Proc. Indian Acad. Sci. Math. Sci. 90 (1981) 239-271.

[49] C. Wilcox, Theory of Bloch waves. J. Anal. Math. 33 (1978) 146-167. 